\title{
Mercury and selenium concentrations in the oysters Saccostrea palmula and Crassostrea corteziensis from four coastal lagoons of NW Mexico: health risk assessment
}

Carlos Humberto Sepúlveda ( $\nabla$ carloshumberto_facimar@uas.edu.mx )

Universidad Autónoma de Sinaloa: Universidad Autonoma de Sinaloa https://orcid.org/0000-00034400-4559

Maria Isabel Sotelo-Gonzalez

Universidad Autónoma de Sinaloa: Universidad Autonoma de Sinaloa

Carmen Cristina Osuna-Martínez

Universidad Autónoma de Sinaloa: Universidad Autonoma de Sinaloa

Manuel García-Ulloa

Instituto Politécnico Nacional: Instituto Politecnico Nacional

Andrés Martín Góngora-Gómez

Instituto Politécnico Nacional: Instituto Politecnico Nacional

Martín Gabriel Frías-Espericueta

Universidad Autónoma de Sinaloa: Universidad Autonoma de Sinaloa

Rebeca Sánchez-Cárdenas

Universidad Autónoma de Sinaloa: Universidad Autonoma de Sinaloa

Laura Gabriela Espinosa-Alonso

Instituto Politécnico Nacional: Instituto Politecnico Nacional

\section{Research Article}

Keywords: Bivalves, Human health, Metal(oid)s, Molar ratio, Northwest Mexico, Spectrophotometry

Posted Date: November 30th, 2021

DOI: https://doi.org/10.21203/rs.3.rs-1062204/v1

License: (9) (1) This work is licensed under a Creative Commons Attribution 4.0 International License.

Read Full License 


\section{Abstract}

Mercury $(\mathrm{Hg})$ and selenium (Se) concentrations in the soft tissue of the oysters Saccostrea palmula and Crassostrea corteziensis from four coastal lagoons (Altata, Macapule, Navachiste, El Colorado) of Northwest Mexico were determined. A total of 2520 specimens of S. palmula and 3780 of C. corteziensis (72.15 \pm 4.95 and $73.57 \pm 5.31 \mathrm{~mm}$, respectively) were sampled seasonally from summer 2019 to spring 2020. The higher $\mathrm{Hg}$ concentration (wet weight) for $S$. palmula $\left(0.13 \pm 0.03-0.11 \pm 0.02 \mu \mathrm{g} \mathrm{g}^{-1}\right)$ and $C$. corteziensis $\left(0.12 \pm 0.02-0.11 \pm 0.03 \mu_{\mathrm{g} \mathrm{g}}{ }^{-1}\right)$ were obtained in summer-autumn 2019; $\mathrm{Hg}$ level was similar among the coastal lagoons and did not exceed the limit established by Mexican legislation and by the Food and Drug Agency. The higher Se concentration occurred in spring 2020 for both oyster species in El Colorado lagoon ( $4.55 \pm 0.02$ and $4.08 \pm 0.05 \mu \mathrm{g} \mathrm{g}^{-1}$, respectively). The Se/ Hg molar ratio of $S$. palmula and $C$. corteziensis ranged from $48.76-149.59$ and 31.72-155.37, respectively; while the Se health benefit value was $19.23-42.28$ and $17.82-35.30$, respectively. The hazard quotient for $\mathrm{Hg}$ estimated as methylmercury- and Se in both species of oyster was below 1 . The high molar ratio obtained $(\mathrm{Se} / \mathrm{Hg}>1)$ indicates that the concentration of Se was sufficient to neutralize the possible toxicity of the $\mathrm{Hg}$, therefore, the consumption of $S$. palmula and $C$. corteziensis from the four coastal lagoons studied does not represent a risk.

\section{Introduction}

Metals and metalloids are pollutants that represent a serious environmental global concern; these elements are characterized by their toxicity, persistence, bioaccumulation, and biomagnification in the ecosystem (Santos et al. 2015; Burioli et al. 2017; Frías-Espericueta et al. 2018). The emission factors of these chemical in the coastal regions of Northwest (NW) Mexico are due to the combination of various natural sources (e.g., geological weathering, volcanic eruptions, forest fires, hydrothermal, and fossil deposits) and anthropogenic activities (e.g., gold mining, coal combustion, deforestation, agriculture, aquaculture, and manufacturing industry) (Páez-Osuna et al. 2017; Bergés-Tiznado et al. 2021). Due to their volatility, mercury $(\mathrm{Hg})$ and selenium $(\mathrm{Se})$ can be mobilized through continental runoff (OsunaMartínez et al. 2010; Ruelas-Inzunza et al. 2011) and atmospheric deposition, which allows them to be even transported to areas far from their main source of origin (Apeti et al. 2012), and, therefore, many organisms are exposed to these elements to some extent (Azad et al. 2019).

In the environment, $\mathrm{Hg}$ is a highly toxic metal without any known biological function; in fact, it is the element responsible of various environmental and human health problems, even at low concentrations (Delgado-Alvarez et al. 2015; Ramos-Osuna et al. 2020), but its organic form (methylmercury, MeHg), is the most toxic (Clarkson et al. 2007; Kakimoto et al. 2019). Among several harmful effects on human health, $\mathrm{Hg}$ causes mitochondrial disorders, lung-kidney damage, myocardial infarction, effects on the central nervous system (mainly in children), cardiovascular atherosclerosis (Ramos-Osuna et al. 2020; Murillo-Cisneros et al. 2021), and can cross the maternal placentas and join the fetal tissues of humans (Ask et al. 2002). 
Selenium is an essential metalloid that plays an important role in the formation of selenocysteine-rich proteins within the cells of most organisms (Stroud et al. 2010; Vega-Sánchez et al. 2020); it also intervenes in brain functions, growth, thyroid hormone metabolism, calcium regulation, and prevents/reverses oxidative stress in humans (Davy and Castellano 2018; Xu et al. 2018; Ralston et al. 2019). Selenium deficiency in human nutrition can lead to infertility (mainly in men) and diseases such as Keshán (heart muscle or myocardial involvement) and Kashin-Beck (osteoarticular involvement) (Yang et al. 2008; Shreenath et al. 2019), while at elevated levels, it can cause selenosis, which is characterized by hair loss, misshapen nails, rashes, swelling of the skin, and severe pain in the extremities (Raymond and Ralston 2004; Ahmad et al. 2021). Recently, it has been documented that, due to its high affinity to form stable non-toxic $\mathrm{Hg} \otimes \mathrm{Se}$ compounds (mercury selenide), without specifically modulating the absorption or excretion of $\mathrm{Hg}$ (Ordiano-Flores et al. 2012; Arcagni et al. 2017; Bergés-Tiznado et al. 2021), the bioavailability of mercury toxicity can be reduced (Bergés-Tiznado et al. 2019; Acosta-Lizárraga et al. 2020; Delgado-Alvarez et al. 2020), when Se/Hg molar ratio is $\mathbb{1} 1$.

Diet (food and water) is considered the main absorption route of essential and toxic elements for humans (Mok et al. 2015). Due to their physicochemical properties, some metals and metalloids can accumulate to high levels through the consumption of certain food, such as shellfish (including oysters) (Zhang and Wong 2007; García-Rico and Tejeda-Valenzuela 2018; Azad et al. 2019). Bivalve mollusks are found in the second trophic level of aquatic ecosystems and represent an important input in cultures and communities that depend on their exploitation and consumption, mainly row (Muñoz-Sevilla et al. 2017). Furthermore, these invertebrates have been widely used as biomonitors in environmental monitoring programs to assess the bioavailability of metals and metalloids (Goldberg et al. 1978; Aguirre-Rubí et al. 2018; Otchere 2019).

The oysters Saccostrea palmula (Carpenter 1857) and Crassostrea corteziensis (Hertlein 1951) are distributed along the Pacific coast from Mexico to Peru (Lodeiros et al. 2020). These ostreids have been used to monitor the Hg concentration on the NW coasts of Mexico (Frías-Espericueta et al. 2009; PáezOsuna and Osuna-Martínez 2015; Ruiz-Fernández et al. 2018), from where they are extracted by local fishermen (Delgado-Alvarez et al. 2015). However, this area is also recognized for its intensive anthropogenic activity (agriculture, mining and aquaculture) (García-Rico and Tejeda-Valenzuela 2018; Jara-Marini et al. 2020), whose wastes are dumped in coastal reservoirs where oysters inhabit; consequently, it leads to a situation of potential risk to human health (Goyer 1997). On the other hand, knowledge on the Se dynamic in both oyster species is scarce. There is only one report that evaluates the presence of Se in the oysters $C$. gigas and $C$. virginica from the San Francisco Bay (California, USA) (Okazaki and Panietz 1981), and no information on the Se/Hg molar ratio in oysters (Scopus 2021) is available. Thus, research related to Se in S. palmula and $C$. corteziensis is necessary to determine its protective effect against mercury toxicity and to establish reference information for comparative studies.

The aim of this study was to determine the total concentrations of mercury and selenium of S. palmula and $C$. corteziensis in four coastal lagoons (Altata, Macapule, Navachiste and El Colorado) of Northwest Mexico, to estimate the human health benefit and risk throught the $\mathrm{Se} / \mathrm{Hg}$ molar ratios, the selenium 
health benefit value $\left(\mathrm{HBV}_{\mathrm{Se}}\right)$, and the hazard quotient $(\mathrm{HQ})$. In addition, the $\mathrm{Hg}$ contamination status of oysters from other lagoons in NW Mexico and other regions of the world was compared. It is hypothesized that the $\mathrm{Hg}$ and Se concentrations of the oysters in this study do not represent a risk for the environment or for human health.

\section{Materials And Methods}

\section{Field sampling}

Oyster samplings were carried out seasonally (summer 2019 to spring 2020) in ten sites distributed within four coastal lagoons of NW Mexico: Altata (AL, three sites), Macapule (ML, two sites), Navachiste (NL, three sites) and El Colorado (ECL, two sites) (Fig. 1). However, oyster species did not coexist at all sampling sites; therefore, $S$. palmula was collected in six sites and $C$. corteziensis in nine. From each site and by species, 105 oysters were selected $(75 \mathrm{for} \mathrm{Hg}$ and Se analysis and $30 \mathrm{for}$ condition index determination, Cl; Muñoz-Sevilla et al. 2017) with a length interval of 60 to $90 \mathrm{~mm}$, to avoid variability in metal content due to differences in specimen size (Sepúlveda et al. 2020).

The oysters were detached from the mangrove roots by hand, placed in metal-free polyethylene bags and transported on ice to the laboratory, where they were cleaned, measured $(\mathrm{mm})$, weighed $(\mathrm{g})$, and chipped (Frías-Espericueta et al. 2018). The $\mathrm{Cl}$ was determined using the equation (1) proposed by Walne and Mann (1975).

$$
\mathrm{CI}=\left(\frac{\text { Soft tissue dry weight }}{\text { Shell dry weight }}\right) \times 1000
$$

\section{Chemical analysis}

Oyster soft tissue samples were lyophilized (Labconco, Kansas City, MO, USA) at low temperature $\left(-85^{\circ} \mathrm{C}\right)$ and high vacuum ( $0.035 \mathrm{mBar}$ ) for $96 \mathrm{~h}$; then, they were ground in a Teflon mortar, homogenized by quartering, and the water content (\%) was determined. Two $\sim 1.0 \mathrm{~g}$ aliquots of each sample were predigested $(\sim 12 \mathrm{~h})$ at room temperature with $10 \mathrm{~mL}$ of concentrated nitric acid $\left(\mathrm{HNO}_{3}\right.$, trace metal grade) in sealed Teflon vials (Savillex, $60 \mathrm{~mL}$ ). Digestion was performed on a heating plate (Barnstead) for $4 \mathrm{~h}$ at $120^{\circ} \mathrm{C}$; subsequently, each sample was diluted with deionized water to a final volume of $50 \mathrm{~mL}$ and stored in polyethylene containers (Delgado-Alvarez et al. 2015; Frías-Espericueta et al. 2018). Total $\mathrm{Hg}$ was determined by atomic absorption spectrophotometry (AAS) coupled to a cold vapor generator (Perkin-Elmer, Analyst 100, coupled MHS 15) and a $253.5 \mathrm{~nm}$ cathode lamp (Muñoz-Sevilla et al. 2017), whereas total Se quantification was analyzed by AAS in a graphite furnace, using a Perkin-Elmer AAnalyst 800 instrument with Zeeman correction effect and a $196 \mathrm{~nm}$ cathode lamp (Acosta-Lizárraga et al. 2020).

\section{Quality assurance/quality control}


The precision of the analytical method and the results were validated by using certified reference material (DOLT-5 ${ }^{\circledR}$; National Research Council Canada, NRCC, USA), with recovery percentages of $116.76 \pm 4.46$ and $90.29 \pm 1.90 \%$ for $\mathrm{Hg}$ and $\mathrm{Se}$ ( $n=10$ for both elements), respectively. Analytical blanks were used to check samples for possible contamination, and all materials used in the sampling and in the laboratory were acid washed (Moody and Lindstrom 1977). The detection limits were 0.002 and $0.013 \mathrm{\mu g} \mathrm{g}^{-1}$ for $\mathrm{Hg}$ and Se, respectively. The coefficient of variation for duplicate samples was $<7.67 \%$. Mercury and Se concentrations are expressed as $\mu \mathrm{g} \mathrm{g}^{-1}$ wet weight ( $w \mathrm{w}, 80.74 \pm 1.60 \%$ moisture content was used for all samples).

\section{$\mathrm{Se} / \mathrm{Hg}$ molar ratio and health benefit}

The Se/Hg molar ratio in the oysters was calculated according to Burger and Gochfeld (2013), dividing the concentration of the element by its molecular weight $\left(78.96\right.$ and $200.59 \mathrm{~g} \mathrm{~mol}^{-1}$ for Se and Hg, respectively). The selenium health benefit value $\left(\mathrm{HBV}_{\mathrm{Se}}\right)$ was calculated using the equation (2) proposed by Ralston et al. (2016).

$$
\mathrm{HBV}_{\mathrm{Se}}=\left(\frac{(\mathrm{Se}-\mathrm{Hg})}{\mathrm{Se}}\right) \times(\mathrm{Se}+\mathrm{Hg})
$$

For the estimation of $\mathrm{HBV}_{\mathrm{Se}}$, the element concentration is presented as $\mu \mathrm{mol} \mathrm{kg}{ }^{-1} \mathrm{ww}$. A positive $\mathrm{HBV}_{\mathrm{Se}}$ value is considered healthy, while a negative value indicates health risks associated with $\mathrm{Hg}$ exposure; the magnitude of the value means the degree of surplus or deficit of selenium, related to the consumption of oysters.

\section{Health risk assessment}

The hazard quotient (HQ) was used to estimate the potential risk to human health due to the level of exposure and/or ingestion of any contaminant. It was calculated for $\mathrm{Hg}$ and Se with the equation (3) proposed by Newman and Unger (2002).

$$
\mathrm{HQ}=\frac{C \times[\text { Weekly intake } / \text { Body weight }]}{\mathrm{RfD}}
$$

where: $C=$ mean concentration of the contaminant $\left(\mu \mathrm{g} \mathrm{g}^{-1}\right)$ in the oysters; $\operatorname{RfD}=$ reference dose of the contaminant ( $\mu \mathrm{g} \mathrm{g}^{-1}$ body weight day $\left.{ }^{-1}\right) ; \mathrm{RfD}_{\mathrm{Hg}}=0.0005$ (FDA 2006); $\mathrm{RfD}_{\mathrm{MeHg}}=0.0001$ (EPA 2001); $\mathrm{RfD}_{\mathrm{Se}}=0.005$ (EPA 1991). The weekly intake of oysters in Mexico is approximately $7.47 \mathrm{~g}$ (CONAPESCA 2017). For the analysis, persons with an mean body weight of $70 \mathrm{~kg}$ (adult men), $60 \mathrm{~kg}$ (adult women), and $16 \mathrm{~kg}$ (children) were included (Alemán-Mateo et al. 2006). Considering that the contribution of MeHg to total $\mathrm{Hg}$ is commonly found between $16.20 \%$ and $62.10 \%$ in oysters (Claisse et al. 2001; Pan and Wang 
2011; Apeti et al. 2012), a conversion of total $\mathrm{Hg}$ to $\mathrm{MeHg}$ was made by adjusting to $39.15 \%$, as proposed by Delgado-Alvarez et al. (2015).

\section{Statistical analyses}

Normality and homoscedasticity of data were analyzed with the Kolmogorov-Smirnov and Bartlett tests, respectively. Comparisons of the biometric data of oysters and average concentrations of $\mathrm{Hg}$ and $\mathrm{Se}$ among the coastal lagoons, sampling sites, and seasons of the year, were assessed by ANOVA's test. Correlations between the concentrations of mercury, selenium, and the molar ratios $(\mathrm{Se} / \mathrm{Hg})$ with the size, total weight, and condition index of oysters were estimated through a Pearson correlation analysis $\left(r_{\mathrm{p}}\right)$. The differences between the content of $\mathrm{Hg}$ and $\mathrm{Se}$ in $\mathrm{S}$. palmula and $C$. corteziensis were detected with the Student's $t$ test for independent variables. The level of significance was $a=0.05$ for all statistical analyzes (Zar 2010), which were performed using the STATISTICA 7 software package (StatSoft, Tulsa, OK, USA).

\section{Results}

\section{Size, total weight, and condition index of oysters}

Size, total weight, and condition index (Cl) of S. palmula $(n=2520)$ and C. corteziensis $(n=3780)$ collected in the four coastal lagoons (NW Mexico), are presented in Table 1. The annual mean value of the shell size in both species did not present significant differences $(p>0.05)$ among the sampling sites. Total weight of $S$. palmula (35.19 $\pm 7.64 \mathrm{~g}$ ) and C. corteziensis ( $49.11 \pm 7.97 \mathrm{~g})$ were significantly higher at the ECL9 and NL7 sites, respectively; while the Cl in both species of oysters was higher in Macapule lagoon (50.23 \pm 17.05 and $51.04 \pm 16.61$ for $S$. palmula and $C$. corteziensis, respectively) $(p<0.05$, Table 1). 
Table 1

Annual mean and standard deviation of size $(\mathrm{mm})$, total weight $(\mathrm{g})$ and condition index of oysters collected in four coastal lagoons in NW Mexico

\begin{tabular}{|c|c|c|c|c|c|c|}
\hline \multirow[t]{2}{*}{ Site } & \multicolumn{2}{|l|}{ Size } & \multicolumn{2}{|c|}{ Total weight } & \multicolumn{2}{|c|}{ Condition index } \\
\hline & Min-Max & Mean \pm SD & Min-Max & Mean \pm SD & Min-Max & Mean \pm SD \\
\hline \multicolumn{7}{|c|}{ Saccostrea palmula } \\
\hline AL1 & $\begin{array}{l}62.63- \\
79.05\end{array}$ & $\begin{array}{l}70.50 \pm \\
2.10\end{array}$ & $\begin{array}{l}21.44- \\
58.74\end{array}$ & $\begin{array}{l}30.41 \pm \\
4.24^{\mathrm{a}}\end{array}$ & $\begin{array}{l}17.07- \\
87.76\end{array}$ & $37.78 \pm 16.25^{a}$ \\
\hline AL2 & $\begin{array}{l}61.59- \\
81.74\end{array}$ & $\begin{array}{l}70.45 \pm \\
3.24\end{array}$ & $\begin{array}{l}21.36- \\
59.20\end{array}$ & $\begin{array}{l}31.72 \pm \\
7.64^{\mathrm{ab}}\end{array}$ & $\begin{array}{l}17.14- \\
86.96\end{array}$ & $\begin{array}{l}43.43 \pm \\
17.18^{\mathrm{ab}}\end{array}$ \\
\hline ML5 & $\begin{array}{l}60.61- \\
80.08\end{array}$ & $\begin{array}{l}72.80 \pm \\
3.41\end{array}$ & $\begin{array}{l}21.60- \\
62.66\end{array}$ & $\begin{array}{l}34.89 \pm \\
7.85^{c}\end{array}$ & $\begin{array}{l}17.24- \\
85.37\end{array}$ & $50.23 \pm 17.05^{c}$ \\
\hline NL6 & $\begin{array}{l}60.14- \\
78.73\end{array}$ & $\begin{array}{l}71.19 \pm \\
4.57\end{array}$ & $\begin{array}{l}21.00- \\
50.05\end{array}$ & $\begin{array}{l}33.38 \pm \\
6.66^{\mathrm{bc}}\end{array}$ & $\begin{array}{l}17.54- \\
77.72\end{array}$ & $\begin{array}{l}42.95 \pm \\
17.85^{\mathrm{ab}}\end{array}$ \\
\hline NL8 & $\begin{array}{l}60.80- \\
84.59\end{array}$ & $\begin{array}{l}72.99 \pm \\
4.24\end{array}$ & $\begin{array}{l}21.20- \\
42.80\end{array}$ & $\begin{array}{l}31.21 \pm \\
6.29^{\mathrm{ab}}\end{array}$ & $\begin{array}{l}19.51- \\
87.53\end{array}$ & $46.21 \pm 17.03^{b}$ \\
\hline ECL9 & $\begin{array}{l}61.21- \\
82.92\end{array}$ & $\begin{array}{l}72.59 \pm \\
2.52\end{array}$ & $\begin{array}{l}21.55- \\
54.70\end{array}$ & $\begin{array}{l}35.19 \pm \\
7.64^{c}\end{array}$ & $\begin{array}{l}19.35- \\
88.57\end{array}$ & $\begin{array}{l}43.31 \pm \\
14.67^{\mathrm{ab}}\end{array}$ \\
\hline Total & NS & $\begin{array}{l}71.75 \pm \\
3.58\end{array}$ & NS & $32.80 \pm 7.05$ & NS & $43.32 \pm 16.63$ \\
\hline \multicolumn{7}{|c|}{ Crassostrea corteziensis } \\
\hline AL2 & $\begin{array}{l}60.17- \\
90.31\end{array}$ & $\begin{array}{l}74.17 \pm \\
4.95\end{array}$ & $\begin{array}{l}27.10- \\
65.10\end{array}$ & $\begin{array}{l}46.08 \pm \\
8.10^{b c}\end{array}$ & $\begin{array}{l}19.34- \\
84.58\end{array}$ & $\begin{array}{l}41.48 \pm \\
16.97^{\mathrm{ab}}\end{array}$ \\
\hline AL3 & $\begin{array}{l}62.40- \\
85.74\end{array}$ & $\begin{array}{l}74.24 \pm \\
3.48\end{array}$ & $\begin{array}{l}26.70- \\
66.50\end{array}$ & $\begin{array}{l}47.18 \pm \\
7.63^{\text {cd }}\end{array}$ & $\begin{array}{l}20.56- \\
86.54\end{array}$ & $\begin{array}{l}47.54 \pm \\
15.43^{b c}\end{array}$ \\
\hline ML4 & $\begin{array}{l}60.63- \\
82.10\end{array}$ & $\begin{array}{l}74.77 \pm \\
3.60\end{array}$ & $\begin{array}{l}32.00- \\
58.60\end{array}$ & $\begin{array}{l}43.16 \pm \\
6.40^{\mathrm{ab}}\end{array}$ & $\begin{array}{l}24.79- \\
87.56\end{array}$ & $51.04 \pm 16.61^{c}$ \\
\hline ML5 & $\begin{array}{l}60.54- \\
88.09\end{array}$ & $\begin{array}{l}72.02 \pm \\
4.51\end{array}$ & $\begin{array}{l}26.90- \\
60.20\end{array}$ & $\begin{array}{l}43.11 \pm \\
7.81^{a}\end{array}$ & $\begin{array}{l}17.09- \\
88.24\end{array}$ & $\begin{array}{l}43.46 \pm \\
18.61^{\mathrm{ab}}\end{array}$ \\
\hline NL6 & $\begin{array}{l}63.76- \\
89.87\end{array}$ & $\begin{array}{l}71.86 \pm \\
3.78\end{array}$ & $\begin{array}{l}31.80- \\
63.40\end{array}$ & $\begin{array}{l}42.71 \pm \\
6.01^{a}\end{array}$ & $\begin{array}{l}17.94- \\
80.65\end{array}$ & $\begin{array}{l}42.03 \pm \\
15.68^{\mathrm{ab}}\end{array}$ \\
\hline
\end{tabular}

Min minimum, Max maximum, $S D$ standard deviation, NS not specified, $A L$ Altata lagoon, $M L$ Macapule lagoon, NL Navachiste lagoon, ECL El Colorado lagoon

Column with different superscript letters denotes significant differences $(p<0.05)$ between the sampling sites and oyster species 


\begin{tabular}{|c|c|c|c|c|c|c|}
\hline \multirow{2}{*}{$\begin{array}{l}\text { Site } \\
\text { NL7 }\end{array}$} & \multicolumn{2}{|l|}{ Size } & \multicolumn{2}{|c|}{ Total weight } & \multicolumn{2}{|c|}{ Condition index } \\
\hline & $\begin{array}{l}60.70- \\
87.20\end{array}$ & $\begin{array}{l}72.81 \pm \\
3.78\end{array}$ & $\begin{array}{l}29.90- \\
68.20\end{array}$ & $\begin{array}{l}49.11 \pm \\
7.97^{\mathrm{d}}\end{array}$ & $\begin{array}{l}22.15- \\
76.92\end{array}$ & $\begin{array}{l}43.25 \pm \\
11.85^{\mathrm{ab}}\end{array}$ \\
\hline NL8 & $\begin{array}{l}63.68- \\
81.02\end{array}$ & $\begin{array}{l}72.13 \pm \\
3.41\end{array}$ & $\begin{array}{l}31.00- \\
57.90\end{array}$ & $\begin{array}{l}43.25 \pm \\
6.01^{\mathrm{ab}}\end{array}$ & $\begin{array}{l}21.05- \\
88.50\end{array}$ & $\begin{array}{l}44.84 \pm \\
16.22^{\mathrm{abc}}\end{array}$ \\
\hline ECL9 & $\begin{array}{l}62.70- \\
91.11\end{array}$ & $\begin{array}{l}72.85 \pm \\
4.39\end{array}$ & $\begin{array}{l}32.10- \\
66.10\end{array}$ & $\begin{array}{l}48.06 \pm \\
8.19^{\mathrm{cd}}\end{array}$ & $\begin{array}{l}17.65- \\
86.42\end{array}$ & $40.68 \pm 14.37^{a}$ \\
\hline ECL10 & $\begin{array}{l}64.69- \\
80.83\end{array}$ & $\begin{array}{l}73.15 \pm \\
2.44\end{array}$ & $\begin{array}{l}31.20- \\
58.40\end{array}$ & $\begin{array}{l}48.07 \pm \\
7.10^{\mathrm{cd}}\end{array}$ & $\begin{array}{l}22.90- \\
88.04\end{array}$ & $\begin{array}{l}46.00 \pm \\
16.66^{\mathrm{abc}}\end{array}$ \\
\hline Total & NS & $\begin{array}{l}73.10 \pm \\
3.95\end{array}$ & NS & $45.64 \pm 7.67$ & NS & $44.48 \pm 16.17$ \\
\hline \multicolumn{7}{|c|}{$\begin{array}{l}\text { Min minimum, Max maximum, } S D \text { standard deviation, } N S \text { not specified, } A L \text { Altata lagoon, } M L \\
\text { Macapule lagoon, } N L \text { Navachiste lagoon, } E C L \text { El Colorado lagoon }\end{array}$} \\
\hline \multicolumn{7}{|c|}{$\begin{array}{l}\text { Column with different superscript letters denotes significant differences }(p<0.05) \text { between the } \\
\text { sampling sites and oyster species }\end{array}$} \\
\hline
\end{tabular}

\section{Mercury And Selenium In The Soft Tissue Of Oysters}

The total $\mathrm{Hg}$ concentration in S. palmula and $C$. corteziensis presented a range between $0.03-0.16$ and $0.02-0.17 \mu \mathrm{g} \mathrm{g}^{-1}$, respectively. In most of the sampling sites, the higher and lower seasonal mean concentrations of $\mathrm{Hg}$ were recorded in summer-autumn 2019 and winter-spring $2020(p<0.05$, Table 2), respectively. In both oyster species, the level of Se presented an order of magnitude higher than those values determined for $\mathrm{Hg}$. The Se content in S. palmula and C. corteziensis showed a seasonal variation, with intervals of $0.99-4.55$ and $0.81-4.08 \mu \mathrm{g} \mathrm{g}^{-1}$, respectively. In both species, the Se showed a tendency to increase seasonally; the lowest concentrations were recorded in summer 2019 and increased in autumn 2019; subsequently, the level of this metaliod increased in winter 2020, and its maximum level was reached in spring $2020(p<0.05$, Table 2$)$. No relationship $(p \otimes 0.05)$ was observed between $\mathrm{Hg}$ and Se with the shell height and total weight of $S$. palmula and $C$. corteziensis. Cl showed correlation with $\mathrm{Hg}$ $\left(r_{p}=-0.29, p<0.05\right)$, but no correlation was found between Se and $\mathrm{Cl}(p \otimes 0.05)$. 
Table 2

Seasonal mean concentrations and standard deviation of total mercury and selenium $\left(\mu \mathrm{g} \mathrm{g}^{-1} \mathrm{ww}\right)$ in the soft tissue of Saccostrea palmula and Crassostrea corteziensis at the sampling sites (NW Mexico)

Site $\quad$ Total $\mathrm{Hg}$

Summer Autumn Winter
Total Se
Spring

Saccostrea palmula

$\begin{array}{lllllllll}\text { AL1 } & 0.15 \pm & 0.13 \pm & 0.09 \pm & 0.07 \pm & 1.34 \pm & 1.96 \pm & 2.87 \pm & 1.70 \pm \\ & 0.03^{\mathrm{c}} & 0.02^{\mathrm{c}} & 0.00^{\mathrm{b}} & 0.00^{\mathrm{a}} & 0.03^{\mathrm{a}} & 0.06^{\mathrm{b}} & 0.02^{\mathrm{c}} & 0.03^{\mathrm{b}} \\ \text { AL2 } & 0.16 \pm & 0.14 \pm & 0.08 \pm & 0.04 \pm & 3.03 \pm & 2.33 \pm & 2.06 \pm & 2.94 \pm \\ & 0.05^{\mathrm{c}} & 0.04^{\mathrm{c}} & 0.02^{\mathrm{b}} & 0.00^{\mathrm{a}} & 0.02^{\mathrm{b}} & 0.02^{\mathrm{a}} & 0.01^{\mathrm{a}} & 0.04^{\mathrm{b}} \\ \text { ML5 } & 0.12 \pm & 0.11 \pm & 0.04 \pm & 0.04 \pm & 1.17 \pm & 1.71 \pm & 2.16 \pm & 1.80 \pm \\ & 0.03^{\mathrm{b}} & 0.02^{\mathrm{b}} & 0.00^{\mathrm{a}} & 0.00^{\mathrm{a}} & 0.03^{\mathrm{a}} & 0.03^{\mathrm{b}} & 0.02^{\mathrm{c}} & 0.03^{\mathrm{b}} \\ \text { NL6 } & 0.07 \pm & 0.08 \pm & 0.05 \pm & 0.03 \pm & 1.98 \pm & 1.79 \pm & 3.03 \pm & 2.86 \pm \\ & 0.02^{\mathrm{c}} & 0.01^{\mathrm{c}} & 0.01^{\mathrm{b}} & 0.00^{\mathrm{a}} & 0.30^{\mathrm{b}} & 0.04^{\mathrm{a}} & 0.23^{\mathrm{c}} & 0.13^{\mathrm{c}} \\ \text { NL8 } & 0.15 \pm & 0.13 \pm & 0.04 \pm & 0.06 \pm & 1.97 \pm & 1.52 \pm & 0.99 \pm & 1.60 \pm \\ & 0.01^{\mathrm{d}} & 0.01^{\mathrm{c}} & 0.00^{\mathrm{a}} & 0.01^{\mathrm{b}} & 0.03^{\mathrm{c}} & 0.03^{\mathrm{b}} & 0.02^{\mathrm{a}} & 0.03^{\mathrm{b}} \\ \text { ECL9 } & 0.13 \pm & 0.10 \pm & 0.03 \pm & 0.05 \pm & 2.33 \pm & 2.88 \pm & 3.60 \pm & 4.55 \pm \\ & 0.02^{\mathrm{d}} & 0.01^{\mathrm{c}} & 0.00^{\mathrm{a}} & 0.01^{\mathrm{b}} & 0.03^{\mathrm{a}} & 0.01^{\mathrm{b}} & 0.02^{\mathrm{c}} & 0.02^{\mathrm{d}}\end{array}$

Crassostrea corteziensis

$\begin{array}{lllllllll}\text { AL2 } & 0.16 \pm & 0.14 \pm & 0.06 \pm & 0.02 \pm & 1.69 \pm & 2.97 \pm & 3.05 \pm & 3.29 \pm \\ & 0.01^{\mathrm{d}} & 0.03^{\mathrm{c}} & 0.00^{\mathrm{b}} & 0.00^{\mathrm{a}} & 0.02^{\mathrm{a}} & 0.04^{\mathrm{b}} & 0.02^{\mathrm{b}} & 0.04^{\mathrm{c}} \\ \text { AL3 } & 0.11 \pm & 0.10 \pm & 0.07 \pm & 0.03 \pm & 1.86 \pm & 1.35 \pm & 2.33 \pm & 3.05 \pm \\ & 0.06^{\mathrm{c}} & 0.04^{\mathrm{c}} & 0.02^{\mathrm{b}} & 0.00^{\mathrm{a}} & 0.03^{\mathrm{b}} & 0.01^{\mathrm{a}} & 0.02^{\mathrm{c}} & 0.02^{\mathrm{d}}\end{array}$

ML4

$\begin{array}{llllllll}0.10 \pm & 0.10 \pm & 0.08 \pm & 0.03 \pm & 0.81 \pm & 2.42 \pm & 2.11 \pm & 1.28 \pm \\ 0.01^{\mathrm{c}} & 0.00^{\mathrm{c}} & 0.01^{\mathrm{b}} & 0.00^{\mathrm{a}} & 0.00^{\mathrm{a}} & 0.13^{\mathrm{c}} & 0.07^{\mathrm{c}} & 0.08^{\mathrm{b}}\end{array}$

ML

$0.09 \pm$

$0.07 \pm$

$0.04 \pm$

$0.02 \pm$

$1.60 \pm$

$1.62 \pm$

$1.53 \pm$

$2.88 \pm$

$0.01^{\mathrm{c}}$

$0.01^{\mathrm{b}}$

$0.00^{\mathrm{a}}$

$0.03^{\mathrm{a}}$

$0.02^{\mathrm{a}}$

$0.02^{\mathrm{a}}$

$0.11^{\mathrm{a}}$

$0.04^{\mathrm{b}}$

NL6

$0.06 \pm$

$0.09 \pm$

$0.06 \pm$

$0.05 \pm$

$1.86 \pm$

$1.51 \pm$

$2.87 \pm$

$3.33 \pm$

$0.01^{\mathrm{c}}$

$0.01^{\mathrm{C}}$

$0.01^{\mathrm{a}}$

$0.00^{\mathrm{b}}$

$0.03^{\mathrm{b}}$

$0.04^{\mathrm{a}}$

$0.02^{\mathrm{c}}$

$0.02^{\mathrm{d}}$

NL7

$0.10 \pm$

$0.11 \pm$

$0.03 \pm$

$0.04 \pm$

$2.94 \pm$

$2.41 \pm$

$2.43 \pm$

$1.17 \pm$

$0.01^{\mathrm{c}}$

$0.01^{\mathrm{c}}$

$0.01^{\mathrm{a}}$

$0.00^{\mathrm{b}}$

$0.07^{\mathrm{C}}$

$0.08^{\mathrm{b}}$

$0.06^{\mathrm{b}}$

$0.04^{\mathrm{a}}$

AL Altata lagoon, $M L$ Macapule lagoon, $N L$ Navachiste lagoon, ECL El Colorado lagoon

For $\mathrm{Hg}$ and $\mathrm{Se}$, rows with different superscript letters denote significant differences $(p<0.05)$ between sampling stations and oyster species 


\begin{tabular}{|c|c|c|c|c|c|c|c|c|}
\hline Site & Total Hg & & & & Total S & & & \\
\hline NL8 & $\begin{array}{l}0.14 \pm \\
0.01^{d}\end{array}$ & $\begin{array}{l}0.15 \pm \\
0.02^{c}\end{array}$ & $\begin{array}{l}0.11 \pm \\
0.01^{\mathrm{a}}\end{array}$ & $\begin{array}{l}0.09 \pm \\
0.02^{b}\end{array}$ & $\begin{array}{l}2.21 \pm \\
0.02^{d}\end{array}$ & $\begin{array}{l}0.90 \pm \\
0.02^{\mathrm{a}}\end{array}$ & $\begin{array}{l}1.08 \pm \\
0.01^{b}\end{array}$ & $\begin{array}{l}1.69 \pm \\
0.04^{c}\end{array}$ \\
\hline ECL9 & $\begin{array}{l}0.12 \pm \\
0.03^{b}\end{array}$ & $\begin{array}{l}0.10 \pm \\
0.02^{b}\end{array}$ & $\begin{array}{l}0.05 \pm \\
0.00^{\mathrm{a}}\end{array}$ & $\begin{array}{l}0.05 \pm \\
0.01^{\mathrm{a}}\end{array}$ & $\begin{array}{l}2.05 \pm \\
0.08^{\mathrm{a}}\end{array}$ & $\begin{array}{l}2.43 \pm \\
0.12^{\mathrm{b}}\end{array}$ & $\begin{array}{l}2.59 \pm \\
0.06^{\mathrm{b}}\end{array}$ & $\begin{array}{l}4.08 \pm \\
0.05^{c}\end{array}$ \\
\hline ECL10 & $\begin{array}{l}0.17 \pm \\
0.04^{d}\end{array}$ & $\begin{array}{l}0.11 \pm \\
0.01^{\mathrm{c}}\end{array}$ & $\begin{array}{l}0.06 \pm \\
0.01^{\mathrm{b}}\end{array}$ & $\begin{array}{l}0.03 \pm \\
0.00^{\mathrm{a}}\end{array}$ & $\begin{array}{l}2.10 \pm \\
0.04^{\mathrm{a}}\end{array}$ & $\begin{array}{l}2.96 \pm \\
0.05^{\mathrm{b}}\end{array}$ & $\begin{array}{l}1.93 \pm \\
0.05^{\mathrm{a}}\end{array}$ & $\begin{array}{l}3.27 \pm \\
0.03^{c}\end{array}$ \\
\hline \multicolumn{9}{|c|}{ AL Altata lagoon, $M L$ Macapule lagoon, $N L$ Navachiste lagoon, ECL El Colorado lagoon } \\
\hline
\end{tabular}

\section{Se/hg Molar Ratio And Health Benefit (Dup: 9 ?)}

No significant differences $(p \otimes 0.05)$ in the mean $\mathrm{Hg}$ level between the sampling sites, were detected in any of studied oyster species (Table 3). On the other hand, Se in S. palmula was significantly $(p<0.05)$ higher in ECL9 $\left(3.34 \pm 0.96 \mu \mathrm{g} \mathrm{g}^{-1}\right)$ while for $C$. corteziensis, in AL2 $\left(2.75 \pm 0.72 \mu \mathrm{g} \mathrm{g}^{-1}\right)$ and ECL9 $(2.79 \pm$ $0.89 \mu \mathrm{g} \mathrm{g}^{-1}$ ) (Table 3$)$. 
Table 3

Annual mean concentrations and standard deviation of total mercury and selenium $\left(\mu \mathrm{g} \mathrm{g}^{-1} \mathrm{ww}\right)$, molar ratio $(\mathrm{Se} / \mathrm{Hg})$ and selenium health benefit value $\left(\mathrm{HBV}_{\mathrm{Se}}\right)$ in the soft tissue of Saccostrea palmula and Crassostrea corteziensis in NW Mexico

\begin{tabular}{|lcccccc|}
\hline Site & Total Hg & $\mu$ mol Hg & Total Se & $\mu$ mol Se & Se/Hg & HBV $_{\text {Se }}$ \\
\hline Saccostrea palmula & & & & & \\
\hline AL1 & $0.11 \pm 0.04$ & $0.55 \pm 0.19$ & $1.97 \pm 0.65^{\mathrm{ab}}$ & $24.92 \pm 8.25$ & 51.30 & 24.90 \\
\hline AL2 & $0.11 \pm 0.06$ & $0.52 \pm 0.28$ & $2.59 \pm 0.47^{\mathrm{b}}$ & $32.81 \pm 5.94$ & 84.94 & 32.80 \\
\hline ML5 & $0.08 \pm 0.04$ & $0.39 \pm 0.22$ & $1.71 \pm 0.41^{\mathrm{ab}}$ & $21.63 \pm 5.18$ & 79.56 & 21.62 \\
\hline NL6 & $0.06 \pm 0.02$ & $0.29 \pm 0.10$ & $2.41 \pm 0.62^{\mathrm{b}}$ & $30.56 \pm 7.89$ & 126.66 & 30.56 \\
\hline NL8 & $0.09 \pm 0.05$ & $0.47 \pm 0.26$ & $1.52 \pm 0.41^{\mathrm{a}}$ & $19.24 \pm 5.13$ & 48.76 & 19.23 \\
\hline ECL9 & $0.08 \pm 0.04$ & $0.40 \pm 0.21$ & $3.34 \pm 0.96^{\mathrm{c}}$ & $42.29 \pm 12.14$ & 149.59 & 42.28 \\
\hline Crassostrea corteziensis & & & & & \\
\hline AL2 & $0.09 \pm 0.07$ & $0.47 \pm 0.33$ & $2.75 \pm 0.72^{\mathrm{c}}$ & $34.82 \pm 9.10$ & 155.37 & 34.81 \\
\hline AL3 & $0.08 \pm 0.04$ & $0.38 \pm 0.19$ & $2.15 \pm 0.72^{\mathrm{b}}$ & $27.21 \pm 9.17$ & 117.69 & 27.20 \\
\hline ML4 & $0.08 \pm 0.03$ & $0.38 \pm 0.17$ & $1.66 \pm 0.74^{\mathrm{ab}}$ & $20.97 \pm 9.41$ & 67.38 & 20.96 \\
\hline ML5 & $0.05 \pm 0.03$ & $0.27 \pm 0.14$ & $1.41 \pm 0.35^{\mathrm{a}}$ & $17.83 \pm 4.45$ & 74.95 & 17.82 \\
\hline NL6 & $0.06 \pm 0.02$ & $0.31 \pm 0.08$ & $2.39 \pm 0.85^{\mathrm{bc}}$ & $30.29 \pm 10.78$ & 107.36 & 30.29 \\
\hline NL7 & $0.07 \pm 0.04$ & $0.35 \pm 0.20$ & $2.24 \pm 0.75^{\mathrm{b}}$ & $28.33 \pm 9.54$ & 103.03 & 28.32 \\
\hline NL8 & $0.12 \pm 0.03$ & $0.61 \pm 0.13$ & $1.47 \pm 0.60^{\mathrm{a}}$ & $18.62 \pm 7.62$ & 31.72 & 18.59 \\
\hline ECL9 & $0.08 \pm 0.04$ & $0.39 \pm 0.19$ & $2.79 \pm 0.89^{\mathrm{c}}$ & $35.31 \pm 11.27$ & 116.74 & 35.30 \\
\hline ECL10 & $0.09 \pm 0.06$ & $0.46 \pm 0.31$ & $2.57 \pm 0.65^{\mathrm{bc}}$ & $32.50 \pm 8.27$ & 124.03 & 32.49 \\
\hline AL Altata lagoon, ML Macapule lagoon, & & & & \\
\hline sampling sites and oyster species & & & & & \\
\hline
\end{tabular}

The Se/Hg molar ratio in the oysters varied among the sampling sites; the lowest value in S. palmula (48.76) and C. corteziensis (31.72) were observed in NL8, while the highest values (149.59 for S. palmula and 155.37 for $C$. corteziensis) were recorded in ECL9 and AL2, respectively (Table 3). No correlations of 
$\mathrm{Se} / \mathrm{Hg}$ were observed between length and total weight in none of the ostreid species $(p>0.05)$. The $\mathrm{Se} / \mathrm{Hg}$ molar ratio of $S$. palmula was correlated with $\mathrm{Cl}\left(r_{\mathrm{p}}=0.30, p \otimes 0.05\right)$. The $\mathrm{HBV}_{\mathrm{Se}}$ obtained for $S$. palmula and $C$. corteziensis indicates that Se concentration found in the two oyster species represents a benefit for human health (Table 3).

\section{Health Risk Assessment}

The HQ value in oysters did not exceed the safe level $(\mathrm{HQ}=1)$ for people with mean body weights of 70 $\mathrm{kg}$ (adult men), $60 \mathrm{~kg}$ (adult women) and $16 \mathrm{~kg}$ (five year-old children) (Table 4). 
Table 4

Hazard quotient (HQ) for $\mathrm{Hg}, \mathrm{MeHg}$ and Se (annual mean concentration; $\mu \mathrm{g} \mathrm{g}^{-1} \mathrm{ww}$ ) of oysters collected in four coastal lagoons of NW Mexico

\begin{tabular}{|c|c|c|c|c|c|c|c|c|c|}
\hline \multirow[t]{2}{*}{ Site } & \multicolumn{3}{|l|}{$\mathrm{Hg}$} & \multicolumn{3}{|c|}{$\mathrm{MeHg}^{\mathrm{a}}$} & \multicolumn{3}{|l|}{ Se } \\
\hline & $M$ & W & C & $M$ & W & C & $M$ & W & C \\
\hline \multicolumn{10}{|c|}{ Saccostrea palmula } \\
\hline AL1 & 0.02 & 0.02 & 0.10 & 0.04 & 0.05 & 0.20 & 0.04 & 0.04 & 0.18 \\
\hline AL2 & 0.02 & 0.02 & 0.09 & 0.04 & 0.05 & 0.19 & 0.05 & 0.06 & 0.24 \\
\hline ML5 & 0.01 & 0.01 & 0.07 & 0.03 & 0.03 & 0.14 & 0.03 & 0.04 & 0.15 \\
\hline NL6 & 0.01 & 0.01 & 0.05 & 0.02 & 0.02 & 0.10 & 0.05 & 0.06 & 0.22 \\
\hline NL8 & 0.02 & 0.02 & 0.08 & 0.03 & 0.04 & 0.17 & 0.03 & 0.03 & 0.14 \\
\hline ECL9 & 0.01 & 0.01 & 0.07 & 0.03 & 0.03 & 0.14 & 0.07 & 0.08 & 0.31 \\
\hline \multicolumn{10}{|c|}{ Crassostrea corteziensis } \\
\hline AL2 & 0.02 & 0.02 & 0.08 & 0.03 & 0.04 & 0.17 & 0.05 & 0.06 & 0.25 \\
\hline AL3 & 0.01 & 0.01 & 0.07 & 0.03 & 0.03 & 0.14 & 0.04 & 0.05 & 0.20 \\
\hline ML4 & 0.01 & 0.01 & 0.07 & 0.03 & 0.03 & 0.13 & 0.03 & 0.04 & 0.15 \\
\hline ML5 & 0.01 & 0.01 & 0.05 & 0.02 & 0.02 & 0.10 & 0.03 & 0.03 & 0.13 \\
\hline NL6 & 0.01 & 0.01 & 0.05 & 0.02 & 0.03 & 0.11 & 0.05 & 0.05 & 0.22 \\
\hline NL7 & 0.01 & 0.01 & 0.06 & 0.02 & 0.03 & 0.12 & 0.04 & 0.05 & 0.20 \\
\hline NL8 & 0.02 & 0.03 & 0.11 & 0.05 & 0.05 & 0.22 & 0.03 & 0.03 & 0.13 \\
\hline ECL9 & 0.01 & 0.01 & 0.07 & 0.03 & 0.03 & 0.14 & 0.05 & 0.06 & 0.26 \\
\hline ECL10 & 0.01 & 0.02 & 0.08 & 0.03 & 0.04 & 0.16 & 0.05 & 0.06 & 0.23 \\
\hline
\end{tabular}

$M$ men, $W$ Women, $C$ Children, $A L$ Altata lagoon, $M L$ Macapule lagoon, $N L$ Navachiste lagoon, $E C L E \mathrm{E}$ Colorado lagoon

${ }^{a}$ Calculated using 0.3915 as mean $\mathrm{MeHg} / \mathrm{Hg}$ ratio, estimated from the ranges reported for oysters by Claisse et al. (2001), Pan and Wang (2011), and Apeti et al. (2012). Total range of MeHg/Hg ratios $0.156-0.621$

\section{Discussion}


The total $\mathrm{Hg}$ in the soft tissue of S. palmula and C. corteziensis ranged from $0.03-0.16$ and $0.02-0.17 \mu \mathrm{g}$ $\mathrm{g}^{-1}$ (wet weight), respectively. In relation to other studies in Mexico, the $\mathrm{Hg}$ concentrations are similar to those obtained in this study (Table 5), but lower than those found for $C$. virginica in the Términos lagoon (Gulf of Mexico), mainly affected by oil, agricultural and urban activities (Aguilar et al. 2012). 
Table 5

Total mercury range concentrations ( $\mu \mathrm{g} \mathrm{g} \mathrm{g}^{-1} \mathrm{ww}$ ) in different oyster species from some coastal lagoons of NW Mexico and other worldwide regions

\begin{tabular}{|c|c|c|c|}
\hline Species & Area (sampling year) & $\begin{array}{l}\text { Total } \\
\mathrm{Hg}\end{array}$ & Author \\
\hline \multicolumn{4}{|c|}{ Coastal lagoons of NW Mexico } \\
\hline C. gigas & Coastal waters, Sonora (1997) & $\begin{array}{l}0.01- \\
0.06\end{array}$ & García-Rico et al. (2001) \\
\hline C. gigas* & Guaymas Bay, Sonora (NA) & $0.04 \star \star$ & Green-Ruiz et al. (2005) \\
\hline $\begin{array}{l}\text { C. } \\
\text { corteziensis* }\end{array}$ & Urías lagoon, Sinaloa (2006) & $\begin{array}{l}0.006- \\
0.01\end{array}$ & Jara-Marini et al. (2008) \\
\hline $\begin{array}{l}\text { C. } \\
\text { corteziensis }\end{array}$ & $\begin{array}{l}\text { Bacochibampo Bay, Sonora (2004- } \\
\text { 2005) }\end{array}$ & $\begin{array}{l}0.03- \\
0.04\end{array}$ & García-Rico et al. (2010) \\
\hline $\begin{array}{l}\text { C. } \\
\text { corteziensis* }\end{array}$ & Coastal lagoons, Sinaloa (NA) & $\begin{array}{l}0.03- \\
0.11\end{array}$ & Osuna-Martínez et al. (2010) \\
\hline C. gigas* & Coastal lagoons, Sinaloa (NA) & $\begin{array}{l}0.01- \\
0.18\end{array}$ & Osuna-Martínez et al. (2010) \\
\hline C. virginica* & Términos lagoon, Campeche (NA) & $\begin{array}{l}0.08- \\
0.40\end{array}$ & Aguilar et al. (2012) \\
\hline C. virginica* & Northern Gulf of Mexico, USA (NA) & $\begin{array}{l}0.006- \\
0.10\end{array}$ & Apeti et al. (2012) \\
\hline $\begin{array}{l}\text { C. } \\
\text { corteziensis* }\end{array}$ & Tobari lagoon, Sonora (2009) & $\begin{array}{l}0.07- \\
0.10\end{array}$ & Jara-Marini et al. (2013) \\
\hline $\begin{array}{l}\text { Crassostrea } \\
\text { spp.* }\end{array}$ & $\begin{array}{l}\text { Coastal lagoons, Sinaloa-Nayarit } \\
(2010-2011)\end{array}$ & $\begin{array}{l}0.02- \\
0.05\end{array}$ & Delgado-Alvarez et al. (2015) \\
\hline S. palmula* & Coastal lagoons, Sinaloa (2008-2009) & $\begin{array}{l}0.04- \\
0.15\end{array}$ & $\begin{array}{l}\text { Páez-Osuna and Osuna- } \\
\text { Martínez (2015) }\end{array}$ \\
\hline $\begin{array}{l}\text { C. } \\
\text { corteziensis* }\end{array}$ & Coastal lagoons, Sinaloa (2008-2009) & $\begin{array}{l}0.03- \\
0.12\end{array}$ & $\begin{array}{l}\text { Páez-Osuna and Osuna- } \\
\text { Martínez (2015) }\end{array}$ \\
\hline C. gigas & La Pitahaya estuary, Sinaloa (2011) & $\begin{array}{l}0.003- \\
0.04\end{array}$ & Góngora-Gómez et al. (2017) \\
\hline C. gigas & $\begin{array}{l}\text { Navachiste lagoon, Sinaloa (2011- } \\
\text { 2012) }\end{array}$ & $\begin{array}{l}0.003- \\
0.03\end{array}$ & Jonathan et al. (2017) \\
\hline C. gigas* & Coastal lagoons, Sinaloa (2013-2014) & $\begin{array}{l}0.03- \\
0.14\end{array}$ & Muñoz-Sevilla et al. (2017) \\
\hline
\end{tabular}

${ }^{*}$ Calculated from dry weight, assuming $80 \%$ moisture **Only the mean value was available. NA not available 


\begin{tabular}{|c|c|c|c|}
\hline Species & Area (sampling year) & $\begin{array}{l}\text { Total } \\
\text { Hg }\end{array}$ & Author \\
\hline $\begin{array}{l}\text { C. } \\
\text { corteziensis* }\end{array}$ & Urías lagoon, Sinaloa (2012-2013) & $\begin{array}{l}0.01- \\
0.05\end{array}$ & Frías-Espericueta et al. (2018) \\
\hline C. gigas & Coastal waters, Sonora (2010) & $\begin{array}{l}0.01- \\
0.13\end{array}$ & $\begin{array}{l}\text { García-Rico and } \\
\text { Tejeda-Valenzuela (2018) }\end{array}$ \\
\hline S. palmula & Coastal lagoons, Sinaloa (2019-2020) & $\begin{array}{l}0.03- \\
0.16\end{array}$ & This study \\
\hline $\begin{array}{l}\text { C. } \\
\text { corteziensis }\end{array}$ & Coastal lagoons, Sinaloa (2019-2020) & $\begin{array}{l}0.02- \\
0.17\end{array}$ & This study \\
\hline \multicolumn{4}{|c|}{ Other worldwide regions } \\
\hline C. gigas* & Minamata Bay, Japan (NA) & $2.00 * \star$ & Eisler (1987) \\
\hline $\begin{array}{l}\text { C. } \\
\text { rhizophorae* }\end{array}$ & $\begin{array}{l}\text { Channel of Santa Cruz, Brazil (1993- } \\
\text { 1994) }\end{array}$ & $\begin{array}{l}0.05- \\
0.44\end{array}$ & Meyer et al. (1998) \\
\hline C. tulipa* & Benya, Ningo lagoons, Ghana (NA) & $\begin{array}{l}0.02- \\
0.04\end{array}$ & Otchere et al. (2003) \\
\hline $\begin{array}{l}\text { C. } \\
\text { rhizophorae* }\end{array}$ & $\begin{array}{l}\text { Gulf of Paria, Venezuela and Trinidad } \\
\text { (NA) }\end{array}$ & $\begin{array}{l}0.002- \\
0.01\end{array}$ & Rojas de Astudillo et al. (2005) \\
\hline C. virginica* & $\begin{array}{l}\text { Gulf of Paria, Venezuela and Trinidad } \\
\text { (NA) }\end{array}$ & $\begin{array}{l}0.002- \\
0.01\end{array}$ & Rojas de Astudillo et al. (2005) \\
\hline $\begin{array}{l}\text { C. } \\
\text { rhizophorae* }\end{array}$ & $\begin{array}{l}\text { Estuarine systems, Northeast Brazil } \\
\text { (NA) }\end{array}$ & $\begin{array}{l}0.004- \\
0.06\end{array}$ & Vaisman et al. (2005) \\
\hline C. gigas & $\begin{array}{l}\text { Oualidia lagoon, Morocco (2004- } \\
\text { 2005) }\end{array}$ & $\begin{array}{l}0.02- \\
0.21\end{array}$ & Maanan (2008) \\
\hline $\begin{array}{l}\text { C. } \\
\text { rhizophorae* }\end{array}$ & Villa Clara, Cuba (NA) & $\begin{array}{l}0.03- \\
0.13\end{array}$ & Olivares-Rieumont et al. (2012) \\
\hline $\begin{array}{l}\text { C. } \\
\text { rhizophorae* }\end{array}$ & $\begin{array}{l}\text { Santos and Paranaguá estuaries, Brazil } \\
(2008-2009)\end{array}$ & $\begin{array}{l}0.02- \\
0.07\end{array}$ & Torres et al. (2012) \\
\hline C. gigas* & $\begin{array}{l}\text { Ebro Delta in Catalonia, Spain (2008- } \\
\text { 2009) }\end{array}$ & $\begin{array}{l}0.02- \\
0.05\end{array}$ & Ochoa et al. (2013) \\
\hline C. gigas & Coastal waters, Korea (2009-2013) & $\begin{array}{l}0.006- \\
0.02\end{array}$ & Mok et al. (2015) \\
\hline C. gigas & Coastal marine ecosystems, Italy (NA) & $\begin{array}{l}0.01- \\
0.34\end{array}$ & Burioli et al. (2017) \\
\hline \multicolumn{4}{|c|}{ *Calculated from dry weight, assuming $80 \%$ moisture } \\
\hline$\star \star$ Only the $\mathrm{n}$ & alue was available. NA not avai & & \\
\hline
\end{tabular}


Compared with other regions of the world, the level of $\mathrm{Hg}$ obtained in S. palmula and C. corteziensis is similar to those reported for $C$. gigas and C. rhizophorae in Morocco (Maanan 2008) and Cuba (OlivaresRieumont et al. 2012), but higher than those determined for $C$. rhizophorae in the Santos and Paranaguá estuary, Brazil (Torres et al. 2012), for C. gigas in the Ebro Delta in Catalonia, Spain (Ochoa et al. 2013) and for $C$. virginica and $C$. rhizophorae in the Gulf of Paria, Venezuela and Trinidad (Rojas de Astudillo et al. 2005). However, the $\mathrm{Hg}$ concentration in this study was lower than those recorded for $C$. gigas in Minamata Bay, Japan (Eisler 1987) and in some coastal and marine regions of Italy where the Japanese oyster is cultivated (Burioli et al. 2017) (Table 5). These differences could be due to the abundance of $\mathrm{Hg}$ in the continental crust (on mean it is higher than $0.056 \mathrm{\mu g} \mathrm{g}^{-1}$ ), since the level of $\mathrm{Hg}$ in the world is not homogeneous and some regions are more enriched than others (Wedepohl 1995; Rytuba 2003). Also, Hg concentration can vary among oyster species due to biological aspects, related to their metabolic activity, differences between sizes, and reproductive period (Páez-Osuna et al. 1995; Frías-Espericueta et al. 2009).

The bioaccumulation of $\mathrm{Hg}$ in S. palmula and $C$. corteziensis could be due to their benthic and feeding habits, since they filter large volumes of seawater (around 10 to $20 \mathrm{~L} \mathrm{~min}^{-1}$ ) through the cilia, mantle and gills (Mazón-Suástegui et al. 2009; Shenai-Tirodkar et al. 2016), where the metal is suspended (Aguilar et al. 2012; Jonathan et al. 2017). Mercury is one of the most persistent chemical elements and can be distributed and accumulated in various tissues and organs of aquatic organisms; eventually, it can be biomagnified from lower trophic levels to humans (Burioli et al. 2017; Murillo-Cisneros et al. 2021). Oysters live mainly attached to the roots of mangroves (i.e. Rhizophora spp.) and on rocky surfaces, exposed and/or buried in muddy sediments (Lodeiros et al. 2020) with a high content of organic matter, which favors retention of metals, such as $\mathrm{Hg}$ (Aguilar et al. 2012; Rangel-Peraza et al. 2015; NavarreteRodríguez et al. 2020). Therefore, it is possible to infer that the sediments in the study area could be one of the most important routes of $\mathrm{Hg}$ accumulation in these bivalves (Sepúlveda et al. 2020).

The annual mean concentration of $\mathrm{Hg}$ in both oyster species did not show significant differences $(p \mathbb{Z}$ 0.05 ) among the sampling sites (Table 3), which could be partially explained by the natural and continuous contribution of leaching from the soils-rocks and by waste from the mining industry (key source of $\mathrm{Hg}$ ). It has been reported that such wastes are transported along rivers and streams that originate in the Sierra Madre Occidental (an area rich in minerals) and, eventually, end up in the coastal lagoons of NW Mexico at low concentration (Murray and Busty 2015; Gamboa-García et al. 2020). During the colonial period until the beginning of the 20th century, the mining industry used approximately $200,000 \mathrm{t}$ of $\mathrm{Hg}$ in the silver and gold extraction process, and it is estimated that just under half of that used $\mathrm{Hg}$ is still present in mining tailings (Delgado-Alvarez et al. 2015; Niane et al. 2019). The Hg used by the mining activity in the state of Sonora (the main gold producer in Mexico), is transported atmospherically to the coastal lagoons of NW Mexico and accumulated in the sediments and different trophic levels (Ruelas-Inzunza et al. 2008); in this way, its impact could reach areas with a low contamination level far from its sources of origin (Apeti et al. 2012; Kakimoto et al. 2019). In addition, $\mathrm{Hg}$ levels in coastal lagoons may increase due to upwelling events and the continuous renewal of seawater 
(Páez-Osuna and Osuna-Martínez 2015). Another important source of this metal is the effluents from intensive agriculture in NW Mexico, since Hg-based fungicides are still used to control pests of various crops (Osuna-Martínez et al. 2010). Mercury can also reach lagoons due to various causes such as: continental runoff, untreated wastewater treatment systems, paint waste, batteries, cement plant waste, and dental objects (Frías-Espericueta et al. 2014).

Regarding the seasonal influence, Osuna-Martínez et al. (2010), Páez-Osuna and Osuna-Martínez (2015) and Muñoz-Sevilla et al. (2017) reported higher concentrations of $\mathrm{Hg}$ in C. gigas, C. corteziensis, and $S$. palmula during the rainy season (summer-autumn), which were related to continental runoff from the vast agricultural areas that reach the coastal lagoons with increased wet deposition. Frías-Espericueta et al. (2018) reported significantly higher concentrations of $\mathrm{Hg}$ in C. corteziensis collected in the Urías lagoon (NW Mexico) in November 2012 (autumn). In this study, a similar trend occurs, since the Hg level in both oyster species was significantly higher $(p<0.05)$ during summer-autumn 2019 (rainy season) (Table 2). On the other hand, García-Rico et al. (2010) did not observe significant differences in the seasonal $\mathrm{Hg}$ concentration in $C$. gigas, probably due to the continuous contribution of anthropogenic activities in Bacochibampo Bay, Sonora.

Despite the natural sources and anthropogenic activities that contribute with $\mathrm{Hg}$ deposition in the coastal lagoons of NW Mexico, the Hg concentration in S. palmula and C. corteziensis was below the maximum permissible limit of 1.0 ( $\mathrm{g} \mathrm{g} \mathrm{g}^{-1}$, wet weight) established by the Mexican legislation (Norma Official Mexicana NOM-031-SSA1-1993), the World Health Organization (WHO 1982), and the United States Food and Drug Agency (FDA 2006), which indicates that the consumption of these species from the sampled lagoons does not represent a risk to human health with respect to $\mathrm{Hg}$ poisoning.

\section{Selenium}

Information about the effect of Se on oysters is scarce. Okazaki and Panietz (1981) reported that the level of this metalloid was higher than that of $\mathrm{Hg}$, in different tissues (mantle, gill and digestive gland) of C. gigas and $C$. virginica, from San Francisco Bay (California, USA), which agrees with the present study. However, the concentration of Se reported by these authors is lower than those determined in S. palmula and $C$. corteziensis (Table 2). This could be due to factors such as the bioavailability of this element in coastal lagoons, as well as the sizes and stages of the organisms (Jara-Marini et al. 2008; Ochoa et al. 2013). The annual mean concentration of Se for S. palmula $\left(3.34 \pm 0.96 \mu \mathrm{g} \mathrm{g}^{-1}\right)$ and C. corteziensis $(2.79$ $\left.\pm 0.89 \mu \mathrm{g} \mathrm{g}^{-1}\right)$ was significantly $(p<0.05)$ higher in ECL9 (Table 3$)$. This site is located in one of the most contaminated areas by metals and metalloids within the El Colorado lagoon (Páez-Osuna and OsunaMartínez 2015), which is part of the Agiabampo-Bacorehuis-Río Fuerte Antiguo lagoon system and, which, receives the contributions of the Fuerte River and the runoff from the highly technical agricultural activities of the municipality of Ahome ( 189,064 ha; SIAP 2019), municipal waste $(\sim 459,310$ inhabitants; INEGI 2020) and shrimp farms ( 12,639 ha; CESASIN 2019) (Cárdenas 2007). NavarroAlarcón and Cabrera-Vique (2008) and Santos et al. (2015) mention that the aquatic environment can be 
contaminated by Se due to agriculture, urban drainage, mining waste, thermoelectric plants, oil refineries and metallic minerals.

In the case of $S$. palmula and $C$. corteziensis collected from the same mangrove roots in sites AL2, ML5, NL6, NL8 and ECL9 (Fig. 1), we hypothesized that the total Hg and Se concentrations were similar between both species, probably because the oysters were under the same water conditions and exposure levels. As expected, there was no significant difference with the concentrations of the study elements between the oysters $(p \otimes 0.05)$. These results may be useful in the intercomparison of $\mathrm{Hg}$ and Se levels among different sites involving S. palmula and C. corteziensis, as mentioned by Páez-Osuna and OsunaMartínez (2015) for the same species and lagoons of sampling. Similar observations were recorded by Osuna-Martínez et al. (2010) for C. gigas and C. corteziensis grown in the Ceuta lagoon, Sinaloa.

\section{Mercury and selenium: influence of the size, weight and condition index in oysters}

According to Trudel and Rasmussen (2006), there is a direct correlation between the $\mathrm{Hg}$ content and the age or body size of aquatic organisms, due to the greater exposure of the metal, which is excreted more slowly as it is ingested. Acosta and Lodeiros (2004) reported that the highest concentrations of metals were proportionally related to the shell size of the clam Tivela mactroides. However, CollaguazoCollaguazo et al. (2017) reported that small clams Anadara tuberculosa have the ability to bioaccumulate a higher concentration of metals (including $\mathrm{Hg}$ ) than large clams. In addition to the species and the level of exposure, other physiological factors such as spawning and body size of bivalves, can influence the accumulation and variability of metals and metalloids in their tissues (Aguilar et al. 2012; Muñoz-Sevilla et al. 2017). In this study, specimens of $S$. palmula and $C$. corteziensis of similar sizes $(72.15 \pm 4.95$ and $73.57 \pm 5.31 \mathrm{~mm}$, respectively; Table 1) were collected to reduce possible variations in the level of metals due to the size of the organisms. However, their body weight varied significantly with the seasons, which suggests that these values could be associated with reproductive cycle, as reported by RodríguezJaramillo et al. (2008) and Góngora-Gómez et al. (2020) for $C$. corteziensis. This pattern has also been observed in other ostreids such as C. rhizophorae (Rebelo et al. 2005), C. gigas and C. corteziensis (Osuna-Martínez et al. 2010), C. virginica (Aguilar et al. 2012), C corteziensis and S. palmula ( $\approx$ Crassostrea palmula) (Páez-Osuna and Osuna-Martínez 2015). In fact, the Hg concentration in S. palmula was slightly correlated with $\mathrm{Cl}\left(r_{\mathrm{p}}=-0.29, p<0.05\right)$, which agrees with that reported by Osuna-Martínez et al. (2010) for $C$. gigas cultivated in the coastal lagoons of NW Mexico.

\section{Se/hg Molar Ratio And Health Benefit}

It has been documented that Se can neutralize the toxic effect of $\mathrm{Hg}$, as long as it's Se/Hg molar ratio is > 1 (Burger and Gochfeld 2013). Selenium intervenes in the Hg demethylation process through the selenocysteine protein, transforming the metal to its inorganic and less toxic form (Escobar-Sánchez et al. 2010; Vega-Sánchez et al. 2020); this way, $\mathrm{Hg}$ can be excreted more easily through pseudofeces and spawning (Rodríguez de la Rua et al. 2005; Havelková et al. 2008). The present study reports for the first time the Se/Hg molar ratio in S. palmula and C. corteziensis in NW Mexico, inclusive, in the distribution 
area of these two species (Pacific coast from Mexico to Peru; Lodeiros et al. 2020). The Se/Hg ratio was always greater than 1 in both species of oyster, which can be explained by the low level of $\mathrm{Hg}$ found (Burger and Gochfeld 2013). These results are similar to the Se/Hg $\otimes 1$ molar ratios found in different groups of organisms, as in shrimps Farfantepenaeus californiensis and Litopenaeus stylirostris (NW of Mexico) (Frías-Espericueta et al. 2016), the mahi mahi fish Coryphaena hippurus (Gulf of California) (Bergés-Tiznado et al. 2019), Pacific hake Merluccius productus (Gulf of California) (Acosta-Lizárraga et al. 2020), crustaceans like the crab Callinectes arcuatus (NW of Mexico) (Delgado-Alvarez et al. 2020), and elasmobranchs like the shark Mustelus henlei (Mexican Pacific Ocean) (Medina-Morales et al. 2020). Regarding the value of the health benefit by Se, calculated through the $\mathrm{HBV}_{\mathrm{Se}}$ index obtained for $S$. palmula and $C$. corteziensis among the sampling sites (Table 3), it indicates a benefit provided by a higher concentration of Se in comparison to $\mathrm{Hg}$ in the soft tissue of oysters. Similar observations were recorded for fishes Thunnus albacares, Vinciguerria lucetia, Lagocephalus lagocephalus, squids Dosidicus gigas, Thysanoteuthis rhombus, Sthenotheuthis oualaniensis and the crab Pleuroncodes planipes $\left(\mathrm{HBV}_{\mathrm{Se}}=64.54,19.41,298.40,12.82,30.30,63.52\right.$ and 13.26, respectively; Ordiano-Flores et al. 2012); as well as for sharks Carcharhinus falciformis $\left(\mathrm{HBV}_{\mathrm{Se}}=52.30\right.$; Bodin et al. 2017), and fish $C$. hippurus $\left(\mathrm{HBV}_{\mathrm{Se}}=1.77\right.$; Vega-Sánchez et al. 2020).

\section{Health Risk Assessment}

Recent studies highlight the importance of evaluating the potential risk to human health from the ingestion of metals and metalloids contained in commercially important fishery products (including oysters). This is done to identify populations at risk, especially in cities and coastal communities dedicated to the capture and production of seafood, where specifically, the annual consumption of oysters is double or more (Delgado-Alvarez 2015) than established as national apparent consumption (0.39 kg/year; CONAPESCA 2017). In the case of the present study, the hazard quotient (HQ) values remained below the risk level $(\mathrm{HQ}<1)$; therefore, the ingestion of $\mathrm{Hg}, \mathrm{MeHg}$ and Se due to the consumption of $S$. palmula and $C$. corteziensis does not represent a risk to human health. These results are similar to the studies by Delgado-Alvarez et al. (2015), Frías-Espericueta et al. (2018), and García-Rico and Tejeda-Valenzuela (2018), who reported HQ values below 1, in Crassostrea spp. (Sonora-Nayarit lagoons), C. corteziensis (Urías lagoon, Sinaloa), and C. gigas (Sonora lagoons), respectively. However, as a precautionary measure, it is important to consider both the frequency of consumption of oysters and the amount of organisms consumed, since Hg levels (above the limits of established legislation) can involve health risks, mainly to vulnerable people (children, pregnant women and/or malnourished people) (Taylor et al. 2014).

\section{Conclusions}

Obtained results indicate that $\mathrm{Hg}$ concentrations in S. palmula and C. corteziensis were similar among the four coastal lagoons (NW of Mexico) and the highest concentrations of this metal occurred in 
summer-autumn 2019, without exceeding the maximum permissible limit of $1.0 \mu \mathrm{g} \mathrm{g}^{-1}$ established by national and international regulations. On the other hand, the total concentrations of Se in both species of oysters were significantly higher in the El Colorado lagoon during spring 2020. No significant differences $(p \otimes 0.05)$ were detected in the levels of $\mathrm{Hg}$ and Se between S. palmula and C. corteziensis, which is explained by sharing the same habitat, implying being exposed to the same water conditions, exposure levels of the elements and feeding on the same resources. The negative correlation observed between $\mathrm{Hg}$ and $\mathrm{Cl}$ in S. palmula, is probably related to its reproductive cycle. The $\mathrm{Se} / \mathrm{Hg}$ molar ratio is reported for the first time in S. palmula and $C$. corteziensis; $\mathrm{Hg}$ toxicity was neutralized by Se, indicating that consumption of raw oysters does not represent a risk.

\section{Declarations}

Ethics approval and consent to participate "Not applicable" in this section.

Consent for publication "Not applicable" in this section.

Availability of data and materials The datasets used and/or analysed during the current study are available from the corresponding author on reasonable request.

Competing interests The authors declare that they have no known competing financial interests or personal relationships that could have appeared to influence the work reported in this paper.

Funding This work was funded by the Secretaría de Investigación y Posgrado (SIP) of the Instituto Politécnico Nacional (IPN) through the projects: SIP 20200526 "Incidencia estacional de nematodos y Perkinsus marinus de la Almeja venus Chione undatella de bahía de La Paz y del Puerto Minero de Santa Rosalía, Baja California Sur, México" and SIP 20200527 "Morfometría y fisiología de la Almeja venus Chione undatella de bahía de La Paz y del Puerto Minero de Santa Rosalía, Baja California Sur, México".

Authors' contributions All authors contributed to the study conception and design. CHS: investigation, sampling, methodology, resources, validation, formal analysis, writing-original draft. MISG and AMGG: resources, sampling and methodology. MGU: conceptualization, funding acquisition, project administration, resources, investigation, formal analysis, writing - review and editing. CCOM: conceptualization, resources, formal analysis, visualization, writing. MGFE, RSC and LGEA: methodology, resources, review and editing. All authors read and approved the final manuscript.

Acknowledgements The authors thank the support from Instituto Politécnico Nacional (IPN), through Secretaría de Investigación y Posgrado, Estímulo al Desempeño de los Investigadores, and Comisión de Operaciones y Fomento de Actividades, and also are grateful with the Sistema Nacional de Investigadores (SNI-CONACYT). Carlos Humberto Sepúlveda and Maria Isabel Sotelo-Gonzalez thank CONACyT for the research fellowship. Thanks to Juan Antonio Hernández Sepúlveda and Dionicio López for participated in the biological sampling. 


\section{References}

1. Acosta V, Lodeiros C (2004) Heavy metals in the clam Tivela mactroides Born, 1778 (Bivalvia: Veneridae) from coastal localities with different degrees of contamination in Venezuela. Cienc Mar 30(2):323-333

2. Acosta-Lizárraga LG, Bergés-Tiznado ME, Bojórquez-Sánchez C, Osuna-Martínez CC, Páez-Osuna $F$ (2020) Bioaccumulation of mercury and selenium in tissues of the mesopelagic fish Pacific hake (Merluccius productus) from the northern Gulf of California and the risk assessment on human health. Chemosphere 255:126941

3. Aguilar CA, Montalvo C, Rodríguez L, Cerón JG, Cerón RM (2012) American oyster (Crassostrea virginica) and sediments as a coastal zone pollution monitor by heavy metals. Int J Environ Sci Technol 9(4):579-586

4. Aguirre-Rubí JR, Luna-Acosta A, Etxebarría N, Soto M, Espinoza F, Ahrens MJ, Marigómez I (2018) Chemical contamination assessment in mangrove-lined Caribbean coastal systems using the oyster Crassostrea rhizophorae as biomonitor species. Environ Sci Pollut Res 25:13396-13415

5. Ahmad S, Bailey EH, Arshad M, Ahmed S, Watts MJ, Stewart AG, Young SD (2021) Environmental and human iodine and selenium status: Lessons from Gilgit-Baltistan, North-East Pakistan. Environ Geochem Health. https://doi.org/10.1007/s10653-021-00943-w

6. Alemán-Mateo H, Salazar G, Hernández-Triana M, Valencia ME (2006) Total energy expenditure, resting metabolic rate and physical activity level in free-living rural elderly men and women from Cuba, Chile and México. Eur J Clin Nutr 60:1258-1265

7. Apeti DA, Lauenstein GG, Evans DW (2012) Recent status of total mercury and methyl mercury in the coastal waters of the Northern Gulf of Mexico using oysters and sediments from NOAA'S mussel watch program. Mar Pollut Bull 64(11):2399-2408. https://doi.org/0.1016/j.marpolbul.2012.08.006

8. Arcagni M, Rizzo A, Juncos R, Pavlin M, Campbell LM, Arribére MA, Horvat M, Guevara SR (2017) Mercury and selenium in the food web of lake Nahuel Huapi, Patagonia, Argentina. Chemosphere 166:163-173

9. Ask K, Åkesson A, Berglund M, Vahter M (2002) Inorganic mercury and methylmercury in placentas of Swedish women. Environ Health Perspect 110:523-526

10. Azad AM, Frantzen S, Bank MS, Nilsen BM, Duinker A, Madsen L, Maage A (2019) Effects of geography and species variation on selenium and mercury molar ratios in Northeast Atlantic marine fish communities. Sci Total Environ 652:1482-1496

11. Bergés-Tiznado ME, Márquez-Farías JF, Osuna-Martínez CC, Torres-Rojas YE, Galván-Magaña F, Páez-Osuna F (2019) Patterns of mercury and selenium in tissues and stomach contents of the dolphinfish Coryphaena hippurus from the SE Gulf of California, Mexico: Concentrations, biomagnification and dietary intake. Mar Pollut Bull 138:84-92

12. Bergés-Tiznado M, Véliz-Hernández I, Bojorquez C, Zamora-García OG, Márquez-Farías JF, PáezOsuna $\mathrm{F}$ (2021) The spotted ratfish Hydrolagus colliei as a potential biomonitor of mercury and 
selenium from deep-waters of the northern Gulf of California. Mar Pollut Bull 164:112102

13. Bodin N, Lesperance D, Albert R, Hollanda S, Michaud P, Degroote M, Churlaud C, Bustamante P (2017) Trace elements in oceanic pelagic communities in the western Indian Ocean.Chemosphere174:354-362

14. Burger J, Gochfeld M (2013) Selenium and mercury molar ratios in commercial fish from New Jersey and Illinois: variation within species and relevance to risk communication. Food Chem Toxicol 57:235-245

15. Burioli EAV, Squadrone S, Stella C, Foglini C, Abete MC, Prearo M (2017) Trace element occurrence in the Pacific oyster Crassostrea gigas from coastal marine ecosystems in Italy. Chemosphere $187: 248-260$

16. Cárdenas GS (2007) Modelación hidrodinámica de la laguna costera El Colorado, Ahome, Sinaloa. Thesis, National Polytechnic Institute (Sinaloa unit), Guasave, Mexico

17. CESASIN (2019) Programa de sanidad en crustáceos. Comité Estatal de Sanidad Acuícola de Sinaloa. https://cesasin.mx/programacrustaceos/. Accessed 20 June 2021

18. Claisse D, Cossa D, Bretaudeau-Sanjuan J, Touchard G, Bombled B (2001) Methylmercury in molluscs along the French coast.Mar Pollut Bull42:329-332

19. Clarkson T, Vyas J, Ballatori N (2007) Mechanisms of mercury disposition in the body. Am J Ind Med 50:755-764

20. Collaguazo-Collaguazo N, Ayala-Armijos H, Machuca-Loja G (2017) Cuantificación de metales pesados en Anadara tuberculosa (Mollusca:bivalvia) del estero Huaylá de Puerto Bolívar, por espectrofotometría de absorción atómica. Ciencia UNEMI 10(24):1-10

21. CONAPESCA (2017) Anuario estadístico de acuacultura y pesca. Comisión Nacional de Acuacultura y Pesca. http://www.Anuario-Estadistico-de-Acuacultura-y-Pesca-2013. Accessed 20 June 2021

22. Davy T, Castellano S (2018) The genomics of selenium: its past. present and future

23. Biochim Biophys Acta 188:2427-2432. https://doi.org/10.1016/j.bbagen.2018.05.020

24. Delgado-Alvarez CG (2015) Mercurio total en recursos pesqueros de importancia comercial en el Noroeste de México: riesgo potencial a la salud humana. Thesis, Autonomous University of Sinaloa, Mazatlán, Mexico

25. Delgado-Alvarez CG, Ruelas-Inzunza J, Osuna-López JI, Voltolina D, Frías-Espericueta MG (2015) Total mercury content in cultured oysters in NW Mexico: health risk assessment. Bull Environ Contam Toxicol 94(2):209-213

26. Delgado-Alvarez CG, Ruelas-Inzunza J, Osuna-Martínez CC, Bergés-Tiznado ME, Escobar-Sánchez O, Ocampo-Rodríguez PO, Soto-Romero KL, Garzón-Raygoza NL, Aguilar-Júarez M, Osuna-López Jl, Frías-Espericueta M (2020) Mercury and selenium concentrations in the crab Callinectes arcuatus from three coastal lagoons of NW Mexico.Environ Sci Pollut Res. https://doi.org/10.1007/s11356020-11396-6 
27. Eisler R (1987) Mercury hazards to fish, wildlife and invertebrates: a synoptic review.US Fish and Wildlife Service

28. EPA (1991) Selenium and Compounds; CASRN 7782-49-2. Integrated Risk Information System, U.S. Environmental Protection Agency. https://cfpub.epa.gov/ncea/iris/iris_documents/documents/subst/0472_summary.pdf. Accessed 15 June 2021

29. EPA (2001) Methylmercury (MeHg); CASRN 22967-92-6. Integrated Risk Information System, U.S. Environmental Protection Agency. https://cfpub.epa.gov/ncea/iris/iris_documents/documents/subst/0073_summary.pdf. Accessed 15 June 2021

30. Escobar-Sánchez O, Galván-Magaña F, Rosíles-Martínez R (2010) Mercury and selenium bioaccumulation in smooth hammerhead shark Sphyrna zygaena Linnaeus from the Mexican Pacific Ocean. Bull Environ Contam Toxicol 84(4):488-491

31. FDA (2006) Mercury levels in commercial fish and shellfish. Washington DC. . Accessed 17 June 2021

32. Frías-Espericueta MG, Osuna-López JI, Bañuelos-Vargas I, López-López G, Muy-Rangel MD, IzaguirreFierro G, Rubio-Carrasco W, Meza-Guerrero PC, Voltolina D (2009) Cadmium, copper, lead and zinc contents of the mangrove oyster, Crassostrea corteziensis, of seven coastal lagoons of NW Mexico. Bull Environ Contam Toxicol 83:595-599

33. Frías-Espericueta MG, Mejía-Cruz R, Osuna-López I, Muy-Rangel MD, Rubio-Carrasco W, AguilarJuárez M, Voltolina D (2014) Metal discharges by Sinaloa rivers to the coastal zone of NW Mexico. Bull Environ Contam Toxicol 92(2):132-136

34. Frías-Espericueta MG, Ramos-Magaña BY, Ruelas-Inzunza J, Soto-Jiménez MF, Escobar-Sánchez O, Aguilar-Juárez M, Izaguirre-Fierro G, Osuna-Martínez CC, Voltolina D (2016) Mercury and selenium concentrations in marine shrimps of NW Mexico: health risk assessment. Environ Monit Assess 188:269

35. Frías-Espericueta MG, Vargas-Jiménez A, Ruelas-Inzunza J, Osuna-López Jl, Aguilar-Juárez M, Bautista-Covarrubias JC, Voltolina D (2018) Total mercury in the mangrove oyster Crassostrea corteziensis of a subtropical lagoon of NW Mexico. Turk J Fish Aquat Sci 18:853-858

36. Gamboa-García DE, Duque G, Cogua P, Marrugo-Negrete JL (2020) Mercury dynamics in macroinvertebrates in relation to environmental factors in a highly impacted tropical estuary: Buenaventura bay, Colombian Pacific. Environ Sci Pollut Res 27:4044-4057

37. García-Rico L, Ramos RE, Vivian J (2001) Determination of total metals in cultivated oysters from the northwest coast of Mexico determined by microwave digestion and atomic absorption spectrometry.J AOAC Int84(6):1909-1913

38. García-Rico L, Tejeda-Valenzuela L, Burgos-Hernández A (2010) Seasonal variations in the concentrations of metals in Crassostrea corteziensis from Sonora, Mexico. Bull Environ Contam Toxicol 85:209-213 
39. García-Rico L, Tejeda-Valenzuela L (2018) Metal concentrations in oysters Crassostrea gigas cultured in the Gulf of California and risk assessment to human health. Expo Health 12:33-39

40. Goldberg ED, Bowen VT, Farrington JW, Harvey G, Martin JH, Parker PL, Risebrough RW, Robertson W, Schneider E, Gamble E (1978) The mussel watch. Environ Conserv 5:101-125

41. Góngora-Gómez AM, García-Ulloa M, Muñoz-Sevilla NP, Domínguez-Orozco AL, Villanueva-Fonseca BP, Hernández-Sepúlveda JA, Ortega-Izaguirre R (2017) Heavy-metal contents in oysters (Crassostrea gigas) cultivated on the Southeast coast of the Gulf of California. Mexico Hidrobiológica 27(2):219227

42. Góngora-Gómez AM, Sepúlveda $\mathrm{CH}$, Verdugo-Escobar HA, Astorga-Castro O, Rodríguez-González H, Domínguez-Orozco AL, Hernández-Sepúlveda JA, García-Ulloa M (2020) Gonadal maturity of Crassostrea corteziensis cultivated in the Gulf of California. Lat Am J Aquat Res 48(3):381-395

43. Goyer AR (1997) Toxic metals and essential metal interactions. Annu Rev Nutr 17:37-50

44. Green-Ruiz C, Ruelas-Inzunza J, Páez-Osuna F (2005) Mercury in surface sediments and benthic organisms from Guaymas Bay, east coast of the Gulf of California. Environ Geochem Health 27:321-329

45. Havelková M, Dušek L, Némethová D, Poleszczuk G, Svobodová Z (2008) Comparison of mercury distribution between liver and muscle-a biomonitoring of fish from lightly and heavily contaminated localities. Sensors 8(7):4095-4109

46. INEGI (2020) Número de habitantes por municipio de Sinaloa. Instituto Nacional de Estadística y Geografía. http://cuentame.inegi.org.mx/monografias/informacion/sin/poblacion/default.aspx? tema $=$ me\&e=25. Accessed 27 October 2021

47. Jara-Marini ME, Soto-Jime'nez MF, Páez-Osuna F (2008) Trace metal accumulation patterns in a mangrove lagoon ecosystem, Mazatlan Harbour, SE Gulf of California. J Environ Sci Health A Tox Hazard Subst Environ Eng 43:1-11

48. Jara-Marini ME, Tapia-Alcaraz JN, Dumer-Gutiérrez A, García-Rico L, García-Hernández J, Páez-Osuna F (2013) Comparative bioaccumulation of trace metals using six filter feeder organisms in a coastal lagoon ecosystem (of the central-east Gulf of California). Environ Monit Assess 185:1071-1085

49. Jara-Marini ME, Molina-García A, Martínez-Durazo A, Páez-Osuna F (2020) Trace metal trophic transference and biomagnification in a semiarid coastal lagoon impacted by agriculture and shrimp aquaculture. Environ Sci Pollut Res 27:5323-5336

50. Jonathan MP, Muñoz-Sevilla NP, Góngora-Gómez AM, Luna-Varela RG, Sujitha SB, Escobedo-Urías DC, Rodríguez-Espinosa PF, Campos-Villegas LE (2017) Bioaccumulation of trace metals in farmed pacific oysters Crassostrea gigas from SW Gulf of California coast, Mexico. Chemosphere 187:311319

51. Kakimoto S, Yoshimitsu M, Akutsu K, Kiyota K, Fujiwara T, Watanabe T, Kajimura K, Yamano T (2019) Concentrations of total mercury and methylmercury in red snow crabs (Chionoecetes japonicus) caught off the coast of Japan. Mar Pollut Bull 145:1-4 
52. Lodeiros C, Valentich-Scott P, Chávez-Villalba J, Mazón-Suástegui JM, Grijalva-Chon JM (2020) Tropical and subtropical ostreidae of the American Pacific: taxonomy, biology, ecology, and genetics. J Shellfish Res 39(2):181-206

53. Maanan M (2008) Heavy metal concentrations in marine molluscs from the Moroccan coastal region. Environ Poll 153(1):176-183

54. Mazón-Suástegui JM, Parres-Haro MA, Ruíz-Ruíz KM, Rodríguez-Jaramillo MC, Saucedo PE (2009) Influence of hatchery diets on early grow-out of the Cortez oyster Crassostrea corteziensis in Guasave, Sinaloa, Mexico. Aquacult Res 40:1908-1914

55. Medina-Morales SA, Corro-Espinosa D, Escobar-Sánchez O, Delgado-Alvarez CG, Ruelas-Inzunza J, Frías-Espericueta MG, Jara-Marini ME, Páez-Osuna F (2020) Mercury (Hg) and selenium (Se) content in the shark Mustelus henlei (Triakidae) in the northern Mexican Pacific. Environ Sci Pollut Res 27(14):16774-16783

56. Meyer U, Hagen W, Medeiros C (1998) Mercury in a northeastern Brazilian mangrove area, a case study: potential of the mangrove oyster Crassostrea rhizophorae as bioindicator for mercury. Mar Biol 131:113-121

57. Mok JS, Yoo HD, Kim PH, Yoon HD, Park YC, Lee TS, Kwon JY, Son KT, Lee HJ, Ha KS, Shim KB, Kim $\mathrm{JH}$ (2015) Bioaccumulation of heavy metals in oysters from the Southern coast of Korea: assessment of potential risk to human health. Bull Environ Contam Toxicol 94:749-755

58. Moody JR, Lindstrom RM (1977) Selection and cleaning of plastic containers for storage of trace element samples.Anal Chem. https://doi.org/10.1021/ac50022a039

59. Muñoz-Sevilla NP, Villanueva-Fonseca BP, Góngora-Gómez AM, García-Ulloa M, Domínguez-Orozco AL, Ortega-Izaguirre R, Campos-Villegas LE (2017) Heavy metal concentrations in diploid and triploid oysters (Crassostrea gigas) from three farms on the north-central coast of Sinaloa, Mexico. Environ Monit Assess 189:536

60. Murillo-Cisneros DA, Zenteno-Savín T, Harley J, Cyr A, Hernández-Almaraz P, Gaxiola-Robles R, Galván-Magaña F, O'Hara TM (2021) Mercury concentrations in Baja California Sur fish: Dietary exposure assessment. Chemosphere 267:129233

61. Murray BP, Busty CJ (2015) Epithermal mineralization controlled by synextensional magmatism in the Guzapares mining district of the Sierra Madre Occidental silicic large igneous province, Mexico. $J$ South Am Earth Sci 58:54-71

62. Navarrete-Rodríguez G, Castañeda-Chávez MR, Lango-Reynoso F (2020) Geoacumulation of heavy metals in sediment of the fluvial-lagoon-deltaic system of the palizada river, Campeche, Mexico. Int J Environ Res Public Health 17(3):969

63. Navarro-Alarcon M, Cabrera-Vique C (2008) Selenium in food and the human body: a review. Sci Total Environ 400:115-141

64. Newman MC, Unger MA (2002) Fundamentals of ecotoxicology. Lewis Publishers

65. Niane B, Guédron S, Feder F, Legros S, Ngom PM, Moritz R (2019) Impact of recent artisanal smallscale gold mining in Senegal: Mercury and methylmercury contamination of terrestrial and aquatic 
ecosystems. Sci Total Environ 669:185-193

66. NOM-031-SSA1- (1993) Bienes y servicios. Productos de la pesca. Moluscos bivalvos frescosrefrigerados y congelados. Especificaciones sanitarias. Diario Oficial de la Federación. Mexico. http://www.dof.gob.mx/nota_detalle.php?codigo=4680717\&fecha=24/03/1994\&print=true. Accessed 11 June 2021

67. Ochoa V, Barata C, Riva MC (2013) Heavy metal content in oysters (Crassostrea gigas) cultured in the Ebro Delta in Catalonia, Spain. Environ Monit Assess 185:6783-6792

68. Okazaki R, Panietz M (1981) Depuration of twelve trace metals in tissues of the oysters Crassostrea gigas and C. virginica. Mar Biol 63:113-120

69. Olivares-Rieumont S, Lima L, Rivero S, Graham DW, Alonso-Hernandez C, Bolaño Y (2012) Mercury levels in sediments and mangrove oysters, Crassostrea rhizophorae from the North coast of Villa Clara, Cuba. Bull Environ Contam Toxicol 88:589-593

70. Ordiano-Flores A, Rosíles-Martínez R, Galván-Magaña F (2012) Biomagnification of mercury and its antagonistic interaction with selenium in yellowfin tuna Thunnus albacares in the trophic web of Baja California Sur, Mexico. Ecotoxicol Environ Saf 86:182-187

71. Osuna-Martínez CC, Páez-Osuna F, Alonso-Rodríguez R (2010) Mercury in cultured oysters (Crassostrea gigas Thunberg, 1793 and C. corteziensis Hertlein, 1951) from four coastal lagoons of the SE Gulf of California, Mexico. Bull Environ Contam Toxicol 85:339-343. https://doi.org/10.1007/s00128-010-0071-4

72. Otchere FA, Joiris CR, Holsbeek L (2003) Mercury in the bivalves Anadara (Senilia) senilis, Perna perna and Crassostrea tulipa from Ghana. Sci Total Environ 304(1-3):369-375

73. Otchere FA (2019) A 50-year review on heavy metal pollution in the environment: Bivalves as biomonitors. Afr J Environ Sci Technol 13(6):220-227

74. Páez-Osuna F, Frías-Espericueta MG, Osuna-López JI (1995) Trace metal concentrations in relation to season and gonadal maturation in the oyster Crassostrea iridescens. Mar Environ Res 40(1):19-31

75. Páez-Osuna F, Osuna-Martínez CC (2015) Bioavailability of cadmium, copper, mercury, lead, and zinc in subtropical coastal lagoons from the southeast Gulf of California using mangrove oysters (Crassostrea corteziensis and Crassostrea palmula). Arch Environ Contam Toxicol 68:305-316

76. Páez-Osuna F, Álvarez-Borrego S, Ruiz-Fernández AC, García-Hernández J, Jara-Marini ME, BergésTiznado ME, Piñón-Gimate A, Alonso-Rodríguez R, Soto-Jiménez MF, Frías-Espericueta MG, RuelasInzunza JR, Green-Ruiz CR, Osuna-Martínez CC, Sánchez-Cabeza JA (2017) Environmental status of the Gulf of California: a pollution review. Earth Sci Rev 166:181-205

77. Pan K, Wang WX (2011) Mercury accumulation in marine bivalves: influences of biodynamics and feeding niche. Environ Pollut 159:2500-2506

78. Ralston NV, Ralston CR, Raymond LJ (2016) Selenium health benefit values: updated criteria for mercury risk assessments. Biol Trace Elem Res 171(2):262-269

79. Ralston NVC, Kaneko JJ, Raymond LJ (2019) Selenium health benefit values provide a reliable index of seafood benefits vs. Risks. J Trace Elem Med Biol 55:50-57 
80. Ramos-Osuna M, Patiño-Mejía C, Ruelas-Inzunza J, Escobar-Sánchez O (2020) Bioaccumulation of mercury in Haemulopsis elongatus and Pomadasys macracanthus from the SE Gulf of California: condition indexes and health risk assessment. Environ Monit Assess 192:704. https://doi.org/10.1007/s10661-020-08599-2

81. Rangel-Peraza JG, de Anda J, González-Farías FA, Rode M, Sanhouse-García A, Bustos-Terrones YA (2015) Assessment of heavy metals in sediment of Aguamilpa Dam, Mexico. Environ Monit Assess 187:134

82. Raymond L, Ralston N (2004) Mercury: selenium interactions and health implications. Seychelles Med Dent J 7(1):72-77

83. Rebelo MF, Amaral MCR, Pfeiffer WC (2005) Oyster condition index in Crassostrea rhizophorae (Guilding, 1828) from a heavy-metal polluted coastal lagoon. Braz J Biol 65(2):345-351

84. de la Rodríguez A, Blasco J, González de Canales ML, Sarasquete C, Arellano JM (2005) Accumulation of copper and histopathological alterations in the oyster Crassostrea angulata. Cienc Mar 31(3): 455-466. https://doi.org/10,7773/cm.v31i3,39

85. Rodríguez-Jaramillo C, Hurtado MA, Romero-Vivas E, Ramírez JL, Manzano M, Palacios E (2008) Gonadal development and histochemistry of the tropical oyster, Crassostrea corteziensis (HERTLEIN, 1951) during an annual reproductive cycle. J Shellfish Res 27(5):1129-1141

86. Rojas de Astudillo L, Chang YI, Bekele I (2005) Heavy metals in sediments, mussels and oysters from Trinidad and Venezuela. Rev Biol Trop 53(1):41-53

87. Ruelas-Inzunza J, Meza-López G, Páez-Osuna F (2008) Mercury in fish that are of dietary importance from the coasts of Sinaloa (SE Gulf of California). J Food Composit Anal 21(3):211-218

88. Ruelas-Inzunza J, Páez-Osuna F, Ruiz-Fernández AC, Zamora-Arellano N (2011) Health risk associated to dietary intake of mercury in selected coastal areas of Mexico. Bull Environ Contam Toxicol 86:180-188

89. Ruiz-Fernández AC, Wu R, Lau TC, Pérez-Bernal LH, Sanchez-Cabeza JA, Chiu JMY (2018) A comparative study on metal contamination in Estero de Urias lagoon, Gulf of California, using oysters, mussels and artificial mussels: Implications on pollution monitoring and public health risk. Environ Poll 243:197-205

90. Rytuba JJ (2003) Mercury from mineral deposits and potential environmental impact. Environ Geol 43:326-338

91. Santos S, Ungureanu G, Boaventura R, Botelho C (2015) Selenium contaminated waters: An overview of analytical methods, treatment options and recent advances in sorption methods. Sci Total Environ $521: 246-260$

92. Scopus (2021) Elsevier Scopus. https://www.elsevier.com/es-mx/solutions/scopus. Accessed 21 June 2021

93. Sepúlveda CH, Góngora-Gómez AM, Pérez-Álvarez S, Rodríguez-González H, Muñoz-Sevilla NP, Villanueva-Fonseca BP, Hernández-Sepúlveda JA, García-Ulloa M (2020) Trace metals in two wild 
populations of the squalid callista clam (Megapitaria squalida) in the southeastern Gulf of California, Mexico. Rev Int Contam Ambie 36(3):667-676

94. Shenai-Tirodkar PS, Gauns MU, Ansari ZA (2016) Concentrations of heavy metals in commercially important oysters from Goa, Central-West coast of India. Bull Environ Contam Toxicol 97:813-819

95. Shreenath AP, Ameer MA, Dooley J (2019) Selenium deficiency. StatPearls Publishing. https://www.ncbi.nlm.nih.gov/books/NBK482260/. Accessed 20 June 2021

96. SIAP (2019) Anuario Estadístico de la Producción Agrícola. Servicio de Información Agroalimentaria y Pesquera. https://nube.siap.gob.mx/cierreagricola/. Accessed 11 June 2021

97. Stroud JL, Broadley MR, Foot I, Fairweather-Tait SJ, Hart DJ, Hurst R, Knott P, Mowat H, Norman K, Scott P, Tucker M, White PJ, McGrath SP, Zhao FJ (2010) Soil factors affecting selenium concentration in wheat grain and the fate and speciation of Se fertilisers applied to soil. Plant Soil 332(12):19-30

98. Taylor DL, Kutil NJ, Malek AJ, Collie JS (2014) Mercury bioaccumulation in cartilaginous fishes from southern New England coastal waters: contamination from a trophic ecology and human health perspective. Mar Environ Res 99:20-33

99. Torres RJ, Cesar A, Pereira CDS, Choueri RB, Abessa DMS, do Nascimento MRL, Fadini PS, Mozeto AA (2012) Bioaccumulation of polycyclic aromatic hydrocarbons and mercury in oysters (Crassostrea rhizophorae) from two Brazilian estuarine zones. Int J Oceanogr 2012:e838320. http://dx.doi.org/10.1155/2012/838320

100. Trudel M, Rasmussen JB (2006) Bioenergetics and mercury dynamics in fish: a modeling perspective. Can J Fish Aquat Sci 63(8):1890-1902

101. Vaisman AG, Marins RV, Lacerda LD (2005) Characterization of the mangrove oyster, Crassostrea rhizophorae, as a biomonitor for mercury in tropical estuarine systems, Northeast Brazil. Bull Environ Contam Toxicol 74:582-588

102. Vega-Sánchez B, Ortega-García S, Ruelas-Inzunza J, Frías-Espericueta M, Escobar-Sánchez O, JaraMarini M (2020) Selenium and mercury in dolphinfish (Coryphaena hippurus) from the Gulf of California: inter-annual variations and selenium health benefit value. Environ Sci Pollut Res 27:2311-2318

103. Walne PR, Mann R (1975) Growth and biochemical composition in Ostrea edulis and Crassostrea gigas. In: Barnes HB (Ed.) Ninth European Marine Biology Symposium. Aberdeen University Press, Aberdeen, pp 587-607

104. Wedepohl KH (1995) The composition of the continental crust.Geochim Cosmochim Acta. https://doi.org/10.1016/0016-7037(95)00038-2

105. WHO (1982) Toxicological evaluation of certain food additives and contaminants. Geneva. http://apps.who.int/iris/handle/10665/41546. Accessed 14 June 2021

106. Xu Z, Regenstein JM, Xie D, Lu W, Ren X, Yuan J (2018) The oxidative stress and antioxidant response of Litopenaeus vannamei to low temperature and air exposure. Fish Shellfish Immunol 72:564-571 
107. Yang DY, Chen YW, Gunn JM, Belzile N (2008) Selenium and mercury in organisms: interactions and mechanisms. Environ Rev. https://doi.org/10.1139/A08-001

108. Zar JH (2010) Biostatistical analysis. Prentice Hall Pearson

109. Zhang L, Wong MH (2007) Environmental mercury contamination in China: Sources and impacts. Environ Int 33:108-121

\section{Figures}
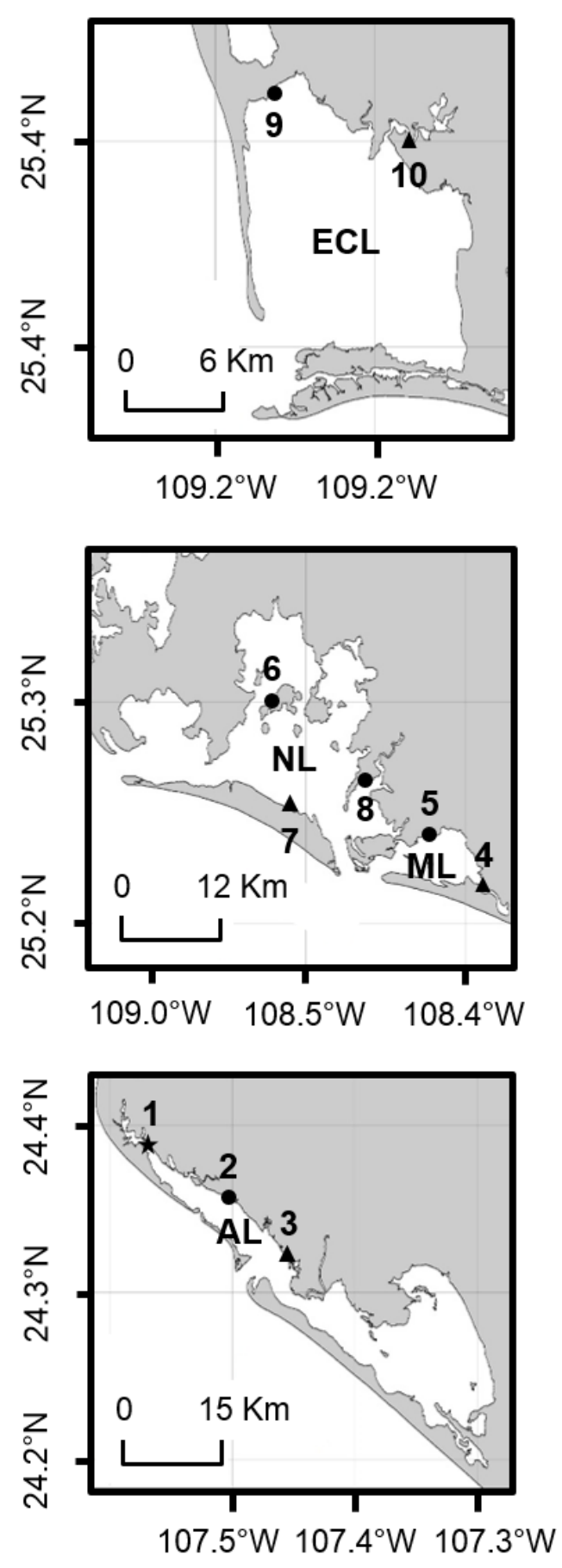

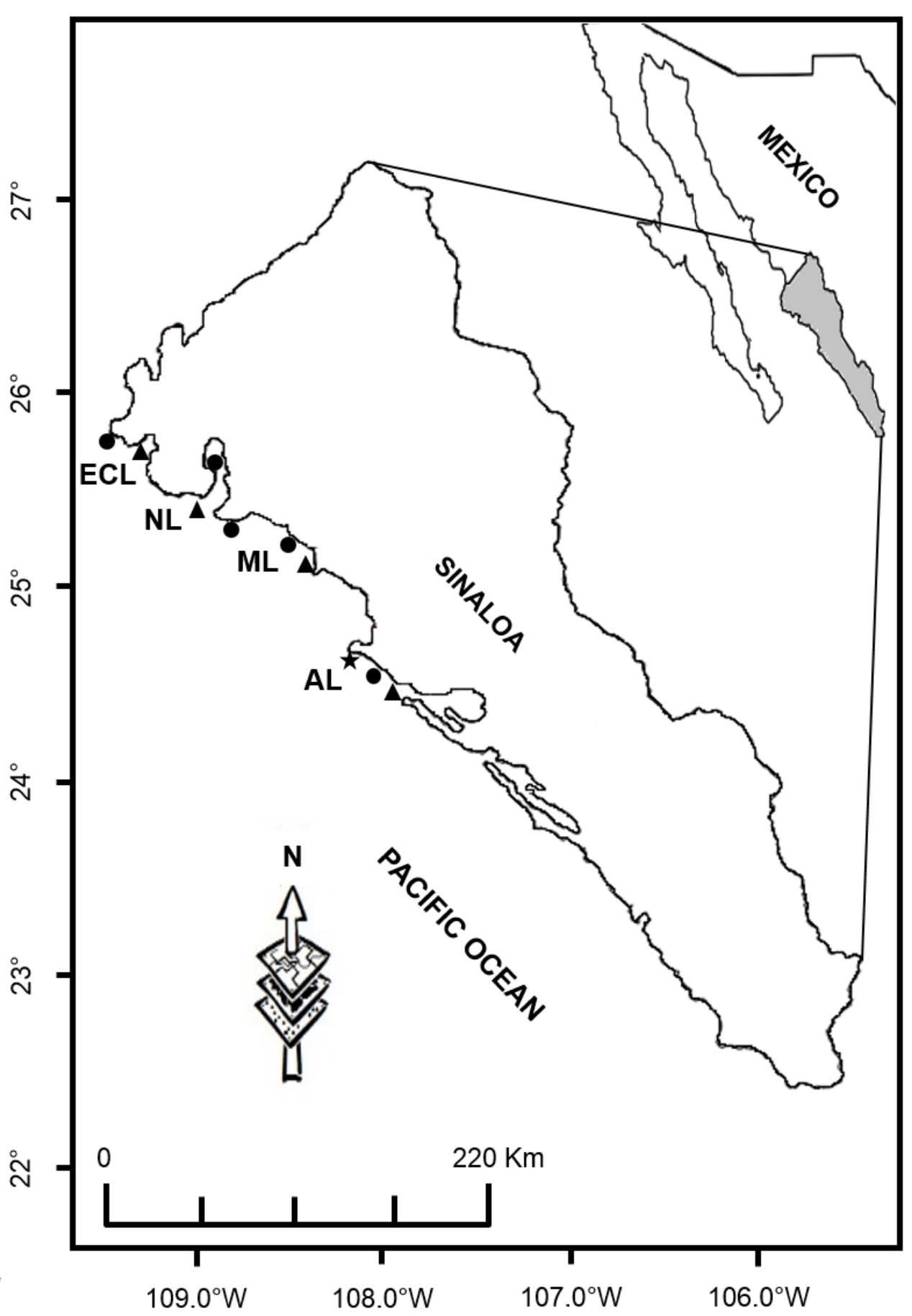

Figure 1 
Location of sampling sites in the coastal lagoons of Northwest Mexico. Saccostrea palmula ( ), Crassostrea corteziensis ( ), both species of oysters ( ). AL Altata lagoon, ML Macapule lagoon, NL Navachiste lagoon, ECL El Colorado lagoon 